\title{
Nanoscale
}

D) Check for updates

Cite this: Nanoscale, 2018, 10, 1162

\section{Particles with similar LET values generate DNA breaks of different complexity and reparability: a high-resolution microscopy analysis of $\gamma \mathrm{H} 2 \mathrm{AX} / 53 \mathrm{BP} 1$ foci}

\author{
Lucie Jezkova, a,b Mariia Zadneprianetc, ${ }^{a, c}$ Elena Kulikova, ${ }^{a, c}$ Elena Smirnova, ${ }^{a}$ \\ Tatiana Bulanova, ${ }^{a, c}$ Daniel Depes, ${ }^{d}$ Iva Falkova, ${ }^{d}$ Alla Boreyko, ${ }^{a, c}$ Evgeny Krasavin, ${ }^{a, c}$ \\ Marie Davidkova, ${ }^{e}$ Stanislav Kozubek, ${ }^{d}$ Olga Valentova ${ }^{\mathrm{b}}$ and Martin Falk (D)*d
}

Biological effects of high-LET (linear energy transfer) radiation have received increasing attention, particularly in the context of more efficient radiotherapy and space exploration. Efficient cell killing by high-LET radiation depends on the physical ability of accelerated particles to generate complex DNA damage, which is largely mediated by LET. However, the characteristics of DNA damage and repair upon exposure to different particles with similar LET parameters remain unexplored. We employed high-resolution confocal microscopy to examine phosphorylated histone $\mathrm{H} 2 \mathrm{AX}(\gamma \mathrm{H} 2 \mathrm{AX}) / \mathrm{p} 53$-binding protein 1 (53BP1) focus streaks at the microscale level, focusing on the complexity, spatiotemporal behaviour and repair of DNA double-strand breaks generated by boron and neon ions accelerated at similar LET values ( 135 $\mathrm{keV} \mu \mathrm{m}^{-1}$ ) and low energies ( 8 and $47 \mathrm{MeV}$ per $\mathrm{n}$, respectively). Cells were irradiated using sharp-angle geometry and were spatially (3D) fixed to maximize the resolution of these analyses. Both high-LET radiation types generated highly complex $\gamma \mathrm{H} 2 \mathrm{AX} / 53 \mathrm{BP} 1$ focus clusters with a larger size, increased irregularity and slower elimination than low-LET $\gamma$-rays. Surprisingly, neon ions produced even more complex $\gamma \mathrm{H} 2 \mathrm{AX} /$ 53BP1 focus clusters than boron ions, consistent with DSB repair kinetics. Although the exposure of cells to $\gamma$-rays and boron ions eliminated a vast majority of foci ( $94 \%$ and $74 \%$, respectively) within $24 \mathrm{~h}, 45 \%$ of the foci persisted in cells irradiated with neon. Our calculations suggest that the complexity of DSB damage critically depends on (increases with) the particle track core diameter. Thus, different particles with similar LET and energy may generate different types of DNA damage, which should be considered in

Received 13th September 2017, Accepted 8th December 2017

DOI: $10.1039 / \mathrm{c} 7 \mathrm{hr} 06829 \mathrm{~h}$

rsc.li/nanoscale future research.

\section{Introduction}

Ionizing radiation (IR) is one of the most effective cytotoxic agents. Due to its ability to ionize atoms or molecules, IR induces several types of DNA damage. Among these lesions, DNA double-strand breaks (DSBs) are the most critical. Cells have evolved sophisticated DNA damage repair systems capable of efficiently removing a vast majority of lesions to maintain genome integrity, which is permanently threatened by various exogenous and endogenous factors. ${ }^{1,2}$

\footnotetext{
${ }^{a}$ Joint Institute for Nuclear Research, Dubna, Russia

${ }^{b}$ University of Chemistry and Technology Prague, Prague, Czech Republic ${ }^{c}$ Dubna State University, Dubna, Russia

${ }^{d}$ Czech Academy of Sciences, Institute of Biophysics, Brno, Czech Republic.

E-mail: falk@ibp.cz; Tel: +420-541517116, +420-728084060

${ }^{e}$ Czech Academy of Sciences, Nuclear Physics Institute, Prague, Czech Republic
}

However, a unique attribute of IR is its ability to deposit energy within nanometre volumes and thus induce clustered/ complex DNA damage containing various combinations of DSBs, single-strand breaks (SSBs), chemically modified or lost bases, DNA-DNA cross-links, DNA-protein cross-links, heatand alkali-labile sites, and other potential impairments. These lesions pose a serious challenge for DNA repair systems, as in many instances, this damage is irreparable, may be repaired incorrectly or may lead to the formation of even more serious DNA damage. ${ }^{1-3}$ Hence, because clustered/complex DNA damage represents the main cause of cell death or mutagenesis upon irradiation, these types of damage have been considered key determinants of the radiobiological effectiveness of radiation.

The complexity of DNA damage depends on the radiation quality, and for radiobiological purposes, this quality is typically described in terms of linear energy transfer (LET). ${ }^{2,4}$ Two main groups of radiation have been distinguished based on 
LET and their mechanism of action and biological effects. Low-LET radiation sparsely and uniformly deposits energy within the whole volume of the cell nucleus. Hence, exposure to $\gamma$-rays or X-rays, two representatives of this category, typically leads to the formation of simple DNA lesions. In contrast, clustered or complex damage (multiple DSBs and other lesions within a few helical DNA turns) is the characteristic feature of high-LET irradiation. ${ }^{1-4}$ The phenomenon of clustered/multiple DNA damage is evoked by the nature of the energy transferred, and by definition, high-LET radiation densely releases energy along the track of the particle such that several types of damage form in a single localized spot volume.

The spatial structure of high-LET particle tracks depends on the physical parameters of the particle and chromatin structure. The distribution of energy deposited by high-LET radiation along the particle track is divided into two spatial components: the core and the penumbra, also known as the region of $\delta$-rays. ${ }^{5,6}$ The core is typically referred as to the central region of extremely dense energy deposition, where biomolecules are directly damaged by the particle itself along with contributions from low-energy knock-on electrons that do not escape from the core volume. Secondary electrons of higher energy ( $\delta$-rays) subsequently radiate out from the core and form the penumbra. Although a generally accepted definition of these track components is not yet available, ${ }^{6}$ the microdosimetric characteristics of the track core and the penumbra together determine the initial complexity of DNA damage. ${ }^{6,7}$ This notion is also true for the relationship between these spatial track/streak components and relative biological effectiveness (RBE) of radiation.

LET is frequently calculated to definitively determine the $\mathrm{RBE}$ of radiation; however, this parameter cannot precisely predict how particle tracks will appear and behave in space and time. A considerable body of evidence has revealed the positive dependence of RBE on LET, where the biological endpoints of irradiation, such as cell killing, ${ }^{8-11}$ induction of mutations ${ }^{12-14}$ (reviewed in Yatagai et $a l^{15}$ ), and formation of chromosomal aberrations, ${ }^{16-19}$ increase as the LET value increases. However, a certain LET value (approximately 100 $\mathrm{keV} \mu \mathrm{m}^{-1}$ ) will correspond to the maximum peak RBE, and from this point, RBE decreases only with increasing LET. Moreover, the most biologically damaging LET value largely fluctuates between 100 and $200 \mathrm{keV} \mu \mathrm{m}^{-1}$ for different particles, depending on the cell type and endpoint studied. ${ }^{7,20-22}$ Hence, a reasonable hypothesis is that the particle generates tracks with different spatiotemporal characteristics according to specific sets of individual physical parameters (e.g., energy, charge, diameter, etc.), even if various combinations of these parameters show similar LET values. ${ }^{7}$ However, experimental studies focusing on the microstructure of DNA damage observed following the action of different high-LET particles with similar LET values are rare.

In addition to initial (static) structure of DNA damage, changes in the DNA dynamics over time after irradiation may also principally influence the reparability of DNA damage and therefore the RBE of radiation types. Chromatin fragmentation occurring in response to high-LET irradiation and chromatin decondensation that occurs during DSB repair (in response to both high-LET and low-LET irradiation) locally mobilizes damaged chromatin to some extent, generating clustered/ complex DNA lesions. ${ }^{23-26}$ The extent of chromatin fragmentation and spatial density of DSBs depend on LET, and the spatiotemporal dynamics of DNA damage is also expected to rely on LET. Nevertheless, researchers have not determined whether and how chromatin dynamics vary for different particles with similar LET. The importance of particle LET/energy becomes particularly relevant for low-dose/particle fluences the conditions addressed in the present study. The characterization of the particle tracks (DNA damage structure) in space and time thus emerges as a promising and necessary new approach to explain many aspects of the radiobiological effects of physically different radiation types.

Studies of spatiotemporal aspects of DNA damage have become feasible with the discovery of the focal accumulation of DSB repair proteins at DSB lesions. ${ }^{27}$ One of the first cell responses to DSB formation is the phosphorylation of histone H2AX on Ser-139, followed by the attraction of numerous downstream repair proteins to sites of DSB. Phosphorylated $\mathrm{H} 2 \mathrm{AX}$ (referred as to $\gamma \mathrm{H} 2 \mathrm{AX}$ ) rapidly spreads over $2 \mathrm{Mbps}$ of damaged chromatin and becomes microscopically detectable as discrete nuclear $(\gamma \mathrm{H} 2 \mathrm{AX})$ foci within minutes after irradiation. $^{27-29}$ Subsequently, numerous repair factors, including p53-binding protein 1 (53BP1), accumulate at $\gamma \mathrm{H} 2 \mathrm{AX}$ foci in a time-dependent manner, resulting in the formation of discrete nuclear domains, which are generally referred to as IR-induced foci (IRIF). ${ }^{23,30}$

Importantly, the structural parameters and assembly and disassembly kinetics of $\gamma \mathrm{H} 2 \mathrm{AX} / \mathrm{IRIF}$ foci reflect the physical characteristics of the damaging agent, chromatin structure, and overall (genetic and physiological) cell status. Microscopy of $\gamma \mathrm{H} 2 \mathrm{AX} / \mathrm{IRIF}$ foci in situ enables complex studies on spatiotemporal aspects of DSB damage induction, repair, and misrepair in the context of intact or even living cells. Moreover, this method offers unprecedented sensitivity and the possibility of simultaneous visualization of multiple DSB repair proteins. ${ }^{30-32}$

The relationship between the number of DSBs and $\gamma \mathrm{H} 2 \mathrm{AX}$ foci is approximately $1: 1$ for low-LET radiation. ${ }^{28,33}$ However, high-LET radiation generates DSBs that are located so close to one another that these lesions cannot be further separated at the resolution of standard optical microscopy. ${ }^{29,34}$ Various super-resolution (nanoscopy) techniques have been proposed and more or less successfully implemented into research practice to overcome this problem ${ }^{35,36}$ (reviewed by Falk $^{37}$ ); however, still these methods remain technically challenging and are typically applicable only to relatively small sample numbers. Two studies by Lorat and co-workers ${ }^{38,39}$ took advantage of transmission electron microscopy to precisely quantify DSB complexity upon high-LET irradiation at a single molecule level. However, despite the superior resolution power and undisputable advantages of this approach, it suffers from serious limitations that primarily result from the complicated sample preparation requirements and the inability to quanti- 
tatively analyse DSB damage in the entire (3D) volume of the cell nucleus. ${ }^{40}$ Hence, a method for the precise, reliable, highthroughput and complex (i.e., in 3D volumes) analysis of DSBs under physiological conditions is not currently available. Many fundamental questions therefore remain open. For example, the above discussed super-resolution study ${ }^{38}$ revealed up to approximately 500 DSBs per $1 \mu^{3}$ volume of the carbon ion track $\left(\mathrm{LET}=190 \mathrm{keV} \mu \mathrm{m}^{-1}\right.$, energy $E=9.5 \mathrm{MeV}$ per n). This result is surprising and prompts questions of how a single DSB is defined at the molecular level and what level of complexity do DSBs generated by different radiation types present.

To our knowledge, the current study represents the first experimental research on the spatiotemporal aspects of DNA damage upon exposure to high-LET radiation with a similar LET and (low) energy. We used high-resolution immunofluorescence confocal microscopy to perform a detailed examination of the DNA damage generated by neon ions, which show potential to improve hadron therapy, ${ }^{41,42}$ and boron ions, which are common secondary particles produced by nuclear interactions between high-energy neutrons and protons. ${ }^{43}$ Surprisingly, only a few experimental ${ }^{6,30,44}$ or theoretical ${ }^{45,46}$ studies (reviewed in Hamada ${ }^{47}$ ) have more or less directly addressed the induction and distribution of DSBs in cell nuclei irradiated with different particles of similar (high) LET. In contrast to these studies, we modified the parameters of the accelerated ions to exhibit similar LET values close to the maximum RBE as well as similar low energies. We focused on lower-energy particles that although they are rarely studied, are attractive for several purposes: they may arise during radiation shielding or affect normal tissue in hadron therapy at the end of the Bragg peak. Moreover, low-energy ions induce more complex and persistent DNA damage than high-energy particles. ${ }^{6,30,48}$

${ }^{20} \mathrm{Ne}$ ions generate DSBs of higher complexity and lower reparability than ${ }^{11} \mathrm{~B}$ ions, despite the similar LET values of both ions and slightly higher energy of neon. Besides other hypotheses, this result may be explained by the morphology and spatiotemporal dynamics of $\gamma \mathrm{H} 2 \mathrm{AX} / 53 \mathrm{BP} 1$ focus streaks that also obviously differed for the two radiation types. Our detailed analysis of these streaks suggests that the particle track structure is the crucial determinant of the RBE of radiation, which roughly depends on LET and energy but might be largely modified by other particle parameters under conditions of similar LET/energy.

\section{Materials and methods}

\subsection{Cell culture}

Primary normal human neonatal dermal fibroblasts, NHDF-Neo (Lonza, CC-2509), were grown in Iscove's Modified Dulbecco's Medium (IMDM) supplemented with 10\% foetal calf serum (FCS) and a 1\% gentamicin-glutamine solution (all reagents from Sigma-Aldrich). Cells were maintained in T-25 cell flasks at $37{ }^{\circ} \mathrm{C}$ in a humidified atmosphere with $5 \% \mathrm{CO}_{2}$. All experiments were performed using cells at passages 6-9.
Prior to irradiation (16-18 h), cells were re-seeded on $14 \mathrm{~mm}$ glass coverslips (glued to the outer side of a $35 \mathrm{~mm}$ Petri dish with a microwell, MatTek Corporation, P35G-0.17014-C) and cultivated to form an $80 \%$ confluent monolayer. For irradiation, dishes were aseptically closed and sealed with Parafilm M (Sigma-Aldrich) to prevent spillage and cell infection. All samples were irradiated at room temperature and immediately returned to the incubator $\left(37^{\circ} \mathrm{C}\right)$.

\subsection{Low-LET and high-LET irradiation}

Non-synchronized cell populations with $>80 \%$ of cells in G1-phase (Fig. 1) were irradiated with accelerated ions using a U-400 M isochronous cyclotron in the Flerov Laboratory of Nuclear Reaction at the Joint Institute for Nuclear Research (JINR, Dubna), and the Genome-M facility was used for the automatic fast irradiation of biological samples. ${ }^{49}$ According to the experiment, cells on glass coverslips (glued to a Petri dish as described above) were irradiated either at a perpendicular $\left(90^{\circ}\right)$ or sharp-angle $\left(10^{\circ}\right)$ geometry, i.e., with $90^{\circ}$ or $10^{\circ}$ angle between the ion beam and the plane of the cell monolayer. The coverslips were oriented towards the ion beam so that the cells were hit by the particles before the beam continued into the culture medium in the Petri dish. Samples that were irradiated in the perpendicular geometry were used to evaluate the kinetics of DSB repair and cell death (apoptosis) upon exposure to radiation. Cells irradiated in the sharp-angle geometry enabled detailed analyses of the morphology of $\gamma \mathrm{H} 2 \mathrm{AX} / 53 \mathrm{BP} 1$ foci and structures of particle tracks (streaks) in space and time. In these experiments, cells were exposed to an average of three particles per nucleus, which corresponds to a dose of $1 \mathrm{~Gy}$ for ${ }^{11} \mathrm{~B}$ ions and $1.2 \mathrm{~Gy}$ for ${ }^{20} \mathrm{Ne}$ ions (calculated as described by Jakob et al. ${ }^{29}$ for the average nuclear area of $186 \mu^{2}$ ). The non-homogeneity within the irradiation field of $14 \mathrm{~mm}$ in diameter was less than $5 \%$, as monitored using five identical flow-type ionization chambers; the central chamber served as the monitor of the radiation dose. ${ }^{49}$

Fibroblasts were exposed to boron or neon beams with a dose rate of approximately 2 Gy $\mathrm{min}^{-1}$. The initial energy of ${ }^{11} \mathrm{~B}$ ions generated by the accelerator was $33 \mathrm{MeV}$ per $\mathrm{n}$. Due to the presence of different absorbers in the path of the ions in route to the sample (e.g., $1.842 \mathrm{~mm}$-thick aluminium foil, $0.170 \mathrm{~mm}$-thick borosilicate glass, etc.), this energy decreased

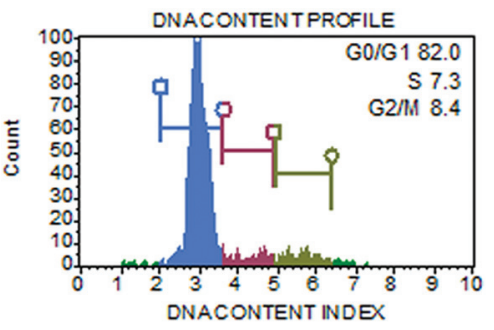

Fig. 1 Illustrative flow cytogram showing the distribution of human skin fibroblasts across the cell cycle at the time of irradiation. The Muse ${ }^{\circledR}$ Cell Analyser (Merck Millipore) and Muse ${ }^{\circledR}$ Cell Cycle Assay Kit (MCH100106) were used according to the manufacturer's instructions. 
to the final value of $8.1 \mathrm{MeV}$ per $\mathrm{n}$ at the plane of the cell monolayer irradiated in the $90^{\circ}$ setup, corresponding to a LET value of $138.1 \mathrm{keV}_{\mu \mathrm{m}}^{-1}$. For the sharp-angle $\left(10^{\circ}\right)$ irradiation geometry, the energy of boron ions was further reduced to 7.5 $\mathrm{MeV}$ per $\mathrm{n}$ (the thickness of the glass at $10^{\circ}$ was $0.979 \mathrm{~mm}$ ) using an additional 1.006 mm-thick aluminium absorber,

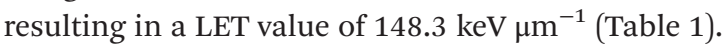

A beam of ${ }^{20} \mathrm{Ne}$ ions accelerated to $50.0 \mathrm{MeV}$ per $\mathrm{n}$ was used as the second type of high-LET radiation. The energy at the cell monolayer was $46.6 \mathrm{MeV}$ per $\mathrm{n}$ in the $90^{\circ}$ geometry and 33.9 $\mathrm{MeV}$ per $\mathrm{n}$ in the sharp-angle $\left(10^{\circ}\right)$ setup; the corresponding LET values were $132.1 \mathrm{keV} \mu \mathrm{m}^{-1}$ and $170.9 \mathrm{keV}_{\mu \mathrm{m}}{ }^{-1}$, respectively. For neon radiation, no additional absorbers (with the exception of mandatory absorbers, e.g., separating foils, electrodes, air, and glass coverslips) were used. The energy and corresponding LET values of ions at the plane of the cell monolayer were calculated using LISE++ software. $^{50}$ The irradiation schemes and radiation characteristics are summarized in Table 1.

Gamma rays from a ${ }^{60} \mathrm{Co}$ source were used as a low-LET radiation. The samples were irradiated at the Rokus-M facility in Dzhelepov Laboratory of Nuclear Problems (JINR, Dubna) with 1 Gy of $\gamma$-rays at a dose rate of $1 \mathrm{~Gy} \mathrm{~min}^{-1}$. Mock-irradiated cells were used as controls in all experiments.

\subsection{Immunofluorescence assay}

Irradiated cells were spatially (3D) fixed at different periods of time post-irradiation (PI), ranging from $5 \mathrm{~min}$ PI to 4 days PI, with $4 \%$ paraformaldehyde/PBS (10 $\mathrm{min}$, room temperature (RT)) after a brief $(2 \times 2 \mathrm{~min})$ wash with PBS (phosphatebuffered saline, $37^{\circ} \mathrm{C}$ ). Fixed cells were quickly rinsed (PBS), washed $3 \times 5$ min with PBS, and permeabilized with $0.2 \%$ Triton-X100/PBS (15 min at RT). After rinsing and washing $(3 \times 5 \mathrm{~min})$ with PBS, cells were treated with the blocking solution I ( $5 \%$ inactivated $\mathrm{FCS} / 2 \%$ bovine serum albumin/PBS) for $30 \mathrm{~min}$ at RT. The primary antibodies - mouse anti- $\gamma \mathrm{H} 2 \mathrm{AX}$ (ab22551, Abcam) and rabbit anti-53BP1 (ab21083, Abcam) were diluted in the blocking solution I (1:700 and 1:600, respectively) and applied to the cells for $10 \mathrm{~min}$ at RT and subsequently overnight at $4^{\circ} \mathrm{C}$. Cells were rinsed with PBS, washed $3 \times 5$ min (RT) with PBS, and blocked (30 min) with blocking solution 2 (5\% inactivated goat serum/PBS) to inhibit nonspecific binding. The secondary antibodies - Texas Red-conjugated goat anti-rabbit (ab6719, Abcam) and FITC-conjugated goat anti-mouse (ab97239, Abcam) - were diluted in blocking solution 2 ( $1: 400$ and $1: 200$, respectively) and applied to the cells for $30 \mathrm{~min}$ (RT, in the dark). This step was followed by washes with PBS (a brief rinse $+3 \times 5 \mathrm{~min}$ at RT), $2 \times$ saline sodium citrate (SSC) $(2 \mathrm{~min}$ ) and $4 \times \mathrm{SSC}+0.1 \%$ Igepal. The chromatin was counterstained with $600 \mathrm{nM}$ 4',6-diamidino-2-phenylindole dihydrochloride (DAPI, Sigma-Aldrich) in Vectashield antifade mounting medium (Vector Laboratories).

\subsection{Image acquisition and experimental data analysis}

Images of the immunofluorescence staining were captured using an automated high-resolution Leica SP5 confocal laser-scanning fluorescence microscopy system equipped with a white laser for the excitation of multiple fluorochromes and sensitive hybrid detectors (Leica). Confocal slices of cells were obtained with a HCX PL APO lambda blue 63.0× 1.40 OIL UV immersion objective with $0.25 \mu \mathrm{m}$ z-step increments across the nuclei, which were 2.7-3.4 $\mu \mathrm{m}$-thick on average. Images were analysed using Acquiarium software, ${ }^{51}$ which enabled the three-dimensional reconstruction of images and inspection of individual $\gamma \mathrm{H} 2 \mathrm{AX}$ and 53BP1 foci in 3D space. For all analyses, only colocalized $\gamma \mathrm{H} 2 \mathrm{AX} / 53 \mathrm{BP} 1$ foci were considered. There were 3 reasons for scoring only the colocalized signals: (1) to increase the precision of DSB detection in the early stages post-irradiation; (2) to increase the probability that unrepaired DSBs are still observed in later and very late periods of time PI; and (3) to partially increase the homogeneity of cells (i.e., the proportion of G1 cells) involved in the analyses. Notably, in early time periods post-irradiation, the $\gamma \mathrm{H} 2 \mathrm{AX}$ signal represents a mixture of small but more developed $\gamma \mathrm{H} 2 \mathrm{AX}$ foci (that colocalize with 53BP1) and similarly small or smaller $\gamma \mathrm{H} 2 \mathrm{AX}$ foci in very early stages, which could sometimes be difficult to distinguish from the background signal. In later periods of time PI, the persistence of the 53BP1 signal at $\gamma \mathrm{H} 2 \mathrm{AX}$ foci allowed us to legitimately presuppose that $\gamma \mathrm{H} 2 \mathrm{AX} /$ 53BP1 foci present in nuclei still represent unrepaired DSBs and are not simply "relics" of previously repaired lesions. Finally, 53BP1 dissociates from $\gamma \mathrm{H} 2 \mathrm{AX}$ foci during late S-phase, ${ }^{52,53}$ G2-phase ${ }^{54}$ and mitosis; ${ }^{54}$ thus, the evaluation of colocalizing $\gamma \mathrm{H} 2 \mathrm{AX}$ foci alone may also increase the predominance of G1 cells in the present analyses.

Table 1 Irradiation schemes and radiation parameters

\begin{tabular}{|c|c|c|c|c|c|c|}
\hline Particle & $Z$ & Geometry $^{\circ}$ & Energy, MeV per $\mathrm{n}$ & LET, $\mathrm{keV} \mu \mathrm{m}^{-1}$ & Fluence $^{*}, 1 \mathrm{~cm}^{-2}$ & Particles per nucleus \\
\hline${ }^{11} \mathrm{~B}$ & 5 & 90 & 8.1 & 138.1 & $4.52 \times 10^{6}$ & 8.4 \\
\hline${ }^{11} \mathrm{~B}$ & 5 & 10 & 7.5 & 148.3 & $4.21 \times 10^{6}$ & 3.3 \\
\hline${ }^{20} \mathrm{Ne}$ & 10 & 90 & 46.6 & 132.1 & $4.73 \times 10^{6}$ & 8.8 \\
\hline${ }^{20} \mathrm{Ne}$ & 10 & 10 & 33.9 & 170.9 & $3.65 \times 10^{6}$ & 2.7 \\
\hline
\end{tabular}

$Z$ - charge, $O$ - geometry of irradiation (the angle between the ion beam and the plane of the cell monolayer), * - fluence per 1 Gy absorbed in water, \# - the number of particles per 1 Gy traversing a nucleus with an average area. The LET of Ne particles increased with increasing distance traversed along the glass at $10^{\circ}$ irradiation, for which it was difficult to compensate. We cannot exclude minor influences on the results, but we consider this effect rather insignificant. Notably, identical conclusions to those drawn using $10^{\circ}$ irradiation were obtained (though with lower precision) using $90^{\circ}$ irradiation, where the LET values for B and Ne ions are much more similar. 
The foci were manually counted by eye ( 2 independent experienced evaluators) in three-dimensional images of approximately 100 nuclei per each experimental condition. In the $10^{\circ}$ irradiation experiments, the software analyses described below supported this estimation. The mean numbers with the standard errors per group were calculated for pooled data from two independent experiments (additional experiments were not possible because of the limited beam time awarded to users and the specific settings required). The area and circularity of $\gamma \mathrm{H} 2 \mathrm{AX} / 53 \mathrm{BP} 1$ foci and complexity of $\gamma \mathrm{H} 2 \mathrm{AX} / 53 \mathrm{BP} 1$ focus clusters were measured in nuclei irradiated in the sharp-angle $\left(10^{\circ}\right)$ geometry, which ensured a sufficiently high microscopic resolution of individual foci. The area and circularity were measured on maximum images composed of all superimposed confocal slices using Adobe Photoshop CS6 Extended software; at least $200 \gamma \mathrm{H} 2 \mathrm{AX} / 53 \mathrm{BP} 1$ foci were scored per group to calculate the mean values with standard errors. The boundaries of individual $\gamma \mathrm{H} 2 \mathrm{AX} / 53 \mathrm{BP} 1$ foci within the cluster and the complexity of $3 \mathrm{D}$ foci in the cluster (number of clustered foci) (Fig. 2) were semi-computationally determined by combining information obtained from (a) the cluster morphology (shape/size) characterized for each individual $0.25 \mu \mathrm{m}$-thick confocal slice of the image $z$-stack; (b) cluster fluorescence intensity heat maps computed for each individual confocal slice of the $z$-stack as well as for the maximum images composed of superimposed confocal slices; and (c) cluster red-channel fluorescence intensity profiles (R-profiles) measured for each individual confocal slice of the image $z$-stack along the path drawn to identify eventual fluorescence maxima and minima between foci in overexposed cluster areas. Since the 53BP1 signals precisely corresponded to $\gamma \mathrm{H} 2 \mathrm{AX}$ signals but presented better mutual separation in clusters, we used the 53BP1 (red) channel for image analyses. First (with the exception of morphologically clearly distinguishable foci), we determined the local fluorescence maxima (fluorescence foci centres; heat maps) of the clusters and compared their numbers per cluster and positions relative to the number and positions of putative foci defined according to cluster morphology. When all parameters were correlated, the number of foci per cluster was counted as the number of fluorescence centres. When two or more foci were identified according to cluster morphology but the number of fluoresce centres was lower (e.g., one large inseparable maximum existed for two putative foci), a fluorescence profile in the red channel (53BP1) was measured along the path intersecting putative foci in more peripheral areas, where the fluorescence signal was not overexposed. Decisions regarding the number of foci were made based on the number of local fluorescence maxima along the path. Alternatively (seemingly one focus contains two fluorescence centres), two foci were only scored under the following conditions: (a) both maxima were intensive, and (b) the focus was large and (c) sufficiently irregular to provide a legitimate impression of the existence of two foci (e.g., large elliptic focus with two intensive eccentrically located fluorescence maxima). The morphology of the clusters was studied in 3D with Acquiarium software, and R-profiles and heat maps were prepared with Image ${ }^{55}$ and CellProfiler ${ }^{56}$ software, respectively. The line demarcating the area of each focus in the cluster (white circles in Fig. 2A) was manually drawn according to the focus heat map signal in the confocal slice showing the strongest fluorescence maximum. Typically, the signal of two levels above the heat map background (green signal in Fig. 2A) was used for this purpose. The described procedures were repeatedly performed for all individual confocal slices in the image $z$-stack, and the data were combined to obtain the final results. The procedure is demonstrated for illustrative foci (later introduced in Fig. 8, Results) generated by $\gamma$-rays, boron ions and neon ions in Fig. 2. Foci forming a spatial unit and connected at least by the "green" fluorescence level (heat maps) were considered to occupy one cluster. Statistical analyses were performed with the Sigma Plot statistical package.

\subsection{Quantification of apoptosis by flow cytometry}

The percentage of apoptotic cells after $\gamma$-ray or boron ion irradiation was determined via flow cytometry staining of Annexin V/7-amino actinomycin (7-AAD)-positive cells (Muse ${ }^{\mathrm{TM}}$ Annexin-V \& Dead Cell Assay kit). Cells that had been irradiated with 1 or 4 Gy were assayed at $24 \mathrm{~h}$ and again at $48 \mathrm{~h}$ PI on the Muse ${ }^{\mathrm{TM}}$ Cell Analyzer, according to the manufacturer's instructions. Briefly, adherent cells and cells floating in the culture medium were collected, diluted to the required concentration $\left(1 \times 10^{5}\right.$ to $5 \times 10^{5}$ cells per $\left.\mathrm{ml}\right)$ and consecutively incubated with the Muse Cell Dispersal Reagent (20 min at RT to prevent the formation of aggregates and clumps) and the Muse $^{\mathrm{TM}}$ Annexin-V \& Dead Cell Assay kit (20 min at RT in the dark). All reagents and the cell analyser were obtained from EMD Millipore Bioscience. Each sample was measured in triplicate, and the mean values with standard errors were calculated. Six thousand cells were acquired and analysed per sample. The proportions of apoptotic cells were determined using the Muse Cell Analyzer software.

\subsection{Core radius calculation}

The radius $r_{\mathrm{c}}$ of the particle track core was calculated based on the model reported by Chatterjee and Holley ${ }^{57}$ using the following formulas:

$$
\begin{gathered}
r_{\mathrm{c}}=\frac{\beta \times c}{\Omega_{\mathrm{p}}}, \\
\Omega_{\mathrm{p}}=\left(\frac{4 \pi n e^{2}}{m}\right)^{1 / 2},
\end{gathered}
$$

where $\beta$ is the velocity of the particle in units of the velocity of light $(c), \Omega_{\mathrm{p}}$ is the plasma oscillation frequency, $n$ is the number density of electrons in water $\left(3 \times 10^{23}\right.$ electrons per $\mathrm{cm}^{3}$ ) and $e$ and $m$ are the charge and mass of the electron, respectively. In water, $\Omega_{\mathrm{p}}=3.09 \times 10^{18} \mathrm{~s}^{-1}$. 

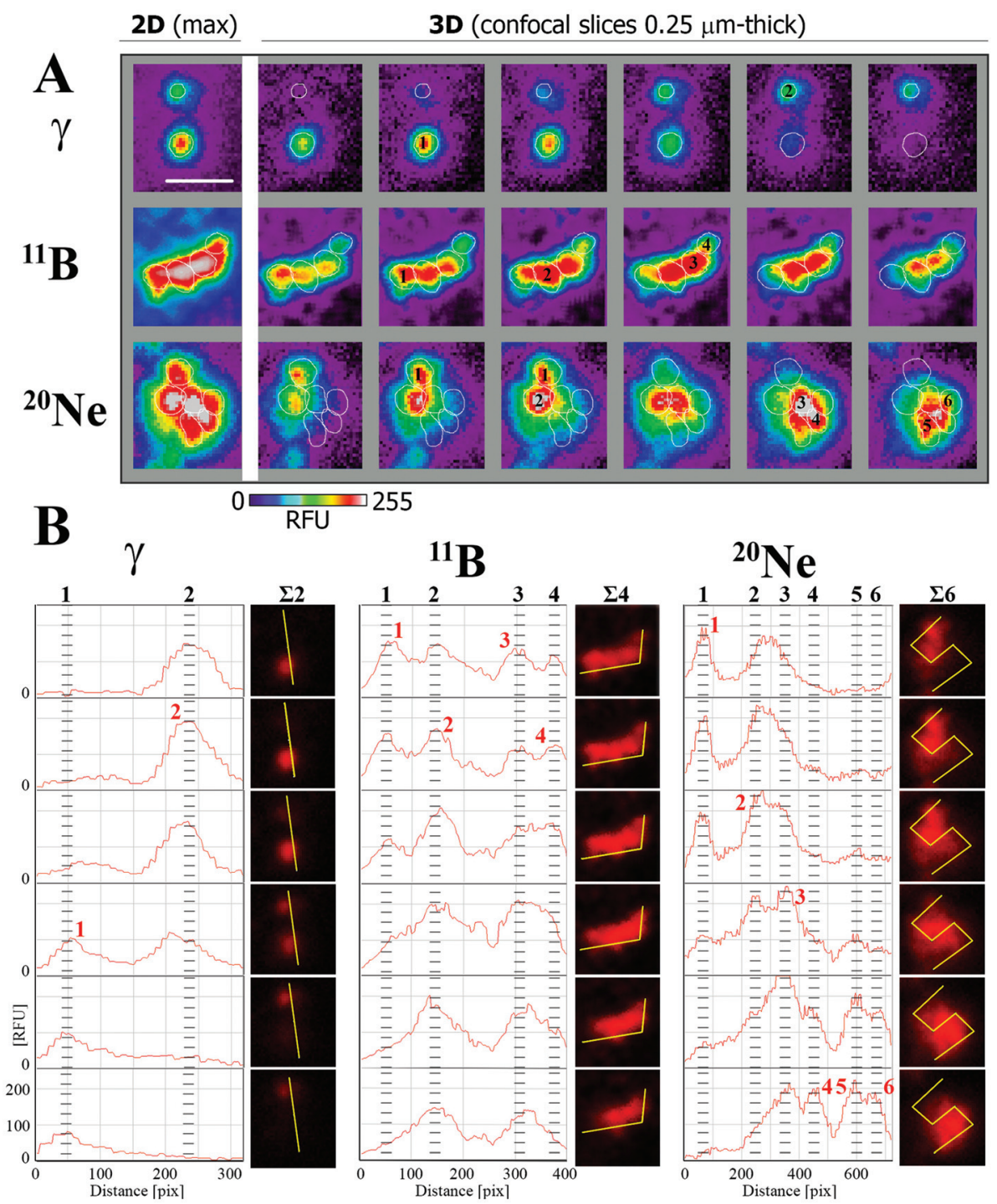

R chanel profiles: RFU (vertical axis) vs pixels along the (yellow; right) trajectory path (horizontal axis)

Fig. 2 Identification and demarcation of individual $\gamma \mathrm{H} 2 \mathrm{AX} / 53 \mathrm{BP} 1$ foci within a cluster, for clusters induced by $\gamma$-rays, boron ions and neon ions. The boundaries of individual $\gamma \mathrm{H} 2 \mathrm{AX} / 53 \mathrm{BP} 1$ foci within the cluster and the complexity of 3D foci in the cluster (number of clustered foci) were semi-computationally determined by combining information obtained from (A) fluorescence intensity heat maps computed for cluster images for each individual confocal slice of the $z$-stack (examples from the second left image to the right) as well as for the maximum images (left) composed of superimposed confocal slices; (B - left panels) red channel fluorescence intensity profiles (R-profiles) measured for each individual confocal slice of the image $z$-stack along the path (yellow line) drawn to identify eventual fluorescence maxima and minima between putative foci (since signals of 53BP1 precisely corresponded to $\gamma \mathrm{H} 2 \mathrm{AX}$ signals but showed better mutual separation in clusters, we used the 53BP1 red channel for image analyses); and (B - right panels) cluster morphology (shape/size) characterized for each individual $0.25 \mu \mathrm{m}$-thick confocal slice of the image $z$-stack. A. For $\gamma$-rays, the heat maps (relative fluorescence intensity units; RFU, 0-255) revealed 2 fluorescence maxima (with red and green intensity levels, respectively), corresponding to 2 morphologically regular and spatially separated foci; no cluster is present. In the case of boron ions, 1 cluster contained 4 maxima of red intensity. The cluster induced by neon ions exhibited 6 fluorescence maxima of red or even white intensity. The lines demarcating the area of each focus in the cluster (white circles) were manually drawn according to the focus heat map signal using the confocal slice showing the strongest fluorescence maximum or on the maximum image. Typically, the signal at two levels above the heat map background (green signal) was used for this purpose. Individual foci are indicated by (black) numbers at the slice of their maximal fluorescence intensity. Scale bar (white) $=$ $400 \mathrm{~nm}$. B. Relative fluorescence intensity (vertical axis, 0-255 [RFU]) profiles for the red (53BP1) channel measured along the path (yellow) shown in the corresponding right panels, where the path is plotted over the analysed clusters of foci. The profiles of consecutive confocal slices are sorted from top to bottom (left column $-\gamma$-rays; middle column $-{ }^{11} \mathrm{~B}$ ions; right column $-{ }^{20} \mathrm{Ne}$ ions). Individual foci are indicated by red numbers at the confocal slice and the position along the yellow path (horizontal axis [pixels]) where they showed the maximum fluorescence intensity. Summarized numbers of foci for all confocal slices are indicated by the black numbers above the top image. As shown in these images, the numbers of foci determined according to R-channel line profiles were well correlated with the results based on the heat maps (A) and cluster morphology analyses (B, right panels). Foci forming a spatial unit and connected by at least the "green" fluorescence level (heat maps) were considered to occupy one cluster. The morphology of the clusters was studied in 3D with Acquiarium software, and R-profiles and heat maps were prepared with ImageJ and CellProfiler software, respectively. 


\section{Results}

3.1 DSB repair kinetics compared for low-LET $\gamma$-rays and two high-LET particles with similar LET values

We examined the kinetics of $\gamma \mathrm{H} 2 \mathrm{AX} / 53 \mathrm{BP} 1$ foci formation and characterized their decrease with time PI in normal human neonatal dermal fibroblasts (NHDF-Neo) exposed to three different types of radiation (section 2.2) to determine how radiation quality affects the induction of double-strand breaks (DSBs) in DNA molecules and assess the ability of human cells to repair this damage. Gamma rays from ${ }^{60} \mathrm{Co}$, representing low-LET radiation, are used in the present study as the standard for comparisons. However, the boron $\left({ }^{11} \mathrm{~B}\right)$ and neon $\left({ }^{20} \mathrm{Ne}\right)$ ions facilitated an examination of the effects of two high-LET particles with comparable LET values (similar to the maximum relative biological efficiency, RBE) and only slightly different energies (Table 1). For kinetics studies, we irradiated cell monolayers with 1 Gy of a particular radiation in the perpendicular $\left(90^{\circ}\right)$ geometry to the beam axis. Consequently, we visualized DSBs using high-resolution immunofluorescence confocal microscopy of colocalization of $\gamma \mathrm{H} 2 \mathrm{AX}$ and 53BP1 foci, the markers of DSBs, ${ }^{58}$ in spatially (3D) fixed cells (Fig. 3). This approach currently provides the maximum sensitivity and fidelity for DSB detection ${ }^{59}$ and, upon reconstructing 3D images of cell nuclei (Fig. 3), also enables efficient discrimination between individual $\gamma \mathrm{H} 2 \mathrm{AX}$ or 53BP1 foci (Fig. 3 and 4). The human skin fibroblasts used in the present study (representing a widely accepted model for studying normal tissue radiosensitivity) ${ }^{60}$ are naturally flat cells with a height of approximately $2.8 \mu \mathrm{m} .{ }^{61}$ We measured highly similar thicknesses of the nuclei of the cells (2.7-3.4 $\mu \mathrm{m}$ ) (Fig. 3), which excluded their significant flattening during 3D fixation. At least 3 closely spaced $\gamma \mathrm{H} 2 \mathrm{AX} / 53 \mathrm{BP} 1$ foci could be distinguished in the $z$-direction in cells irradiated with ${ }^{11} \mathrm{~B}$ or ${ }^{20} \mathrm{Ne}$ ions. Hence, we were able to precisely examine the qualitative and quantitative characteristics of chromatin damage and DSB repair following exposure to different radiation types. The $\gamma \mathrm{H}$ AX/53BP1 detection - 2D analysis vs. 3D analysis

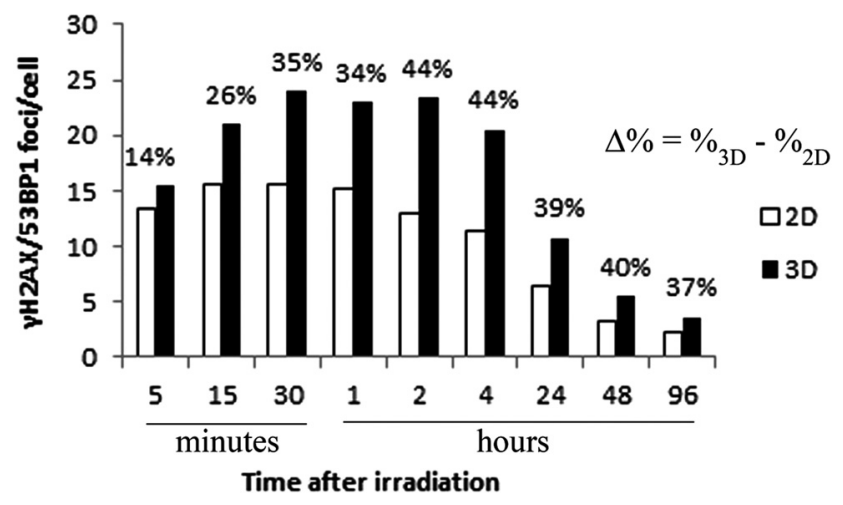

Fig. 4 Differences between the 2D (white columns) and detailed 3D (black columns) quantification of $\gamma \mathrm{H} 2 \mathrm{AX} / 53 \mathrm{BP} 1 \mathrm{foci}$ in NHDF-Neo fibro-

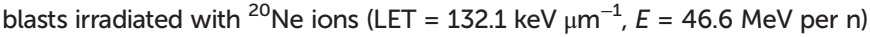
and spatially (3D) fixed with paraformaldehyde in both cases. The maximum images superimposed from individual confocal slices (through the complete volume of the nucleus) were used in 2D analyses, whereas these slices were individually inspected in 3D analyses. The means (left axis) \pm standard errors for pooled data from two experiments (100 counted cells) and the percentage underestimation of the number of foci in the 2D analysis (numbers above the pairs of bars) are indicated.

enhancement of DSB resolution in the 3D analysis compared with the $2 \mathrm{D}$ analysis is quantified in Fig. 4.

We quantified the colocalization of $\gamma \mathrm{H} 2 \mathrm{AX}$ and 53BP1 foci at numerous times PI, ranging from $5 \mathrm{~min}$ PI to $96 \mathrm{~h}$ PI, to evaluate the kinetics of DSB repair. The maximum number of individual radiation-induced $\gamma \mathrm{H} 2 \mathrm{AX} / 53 \mathrm{BP} 1$ foci per cell was observed between $30 \mathrm{~min}$ PI and $1 \mathrm{~h}$ PI, and the value was higher for samples exposed to both types of accelerated ions $\left({ }^{11} \mathrm{~B}: 24.0 \pm 1.1,{ }^{20} \mathrm{Ne}: 23.9 \pm 1.3\right)$ than for samples exposed to $\gamma$-rays $(20.1 \pm 0.5)$ (Fig. 5).

Additionally, the dynamics of foci formation and elimination differed with the radiation type. The fastest production
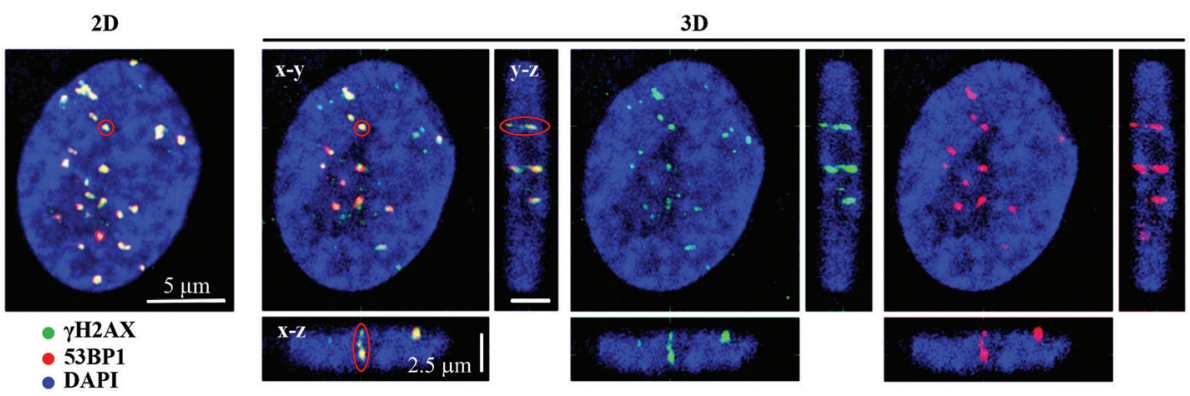

Fig. 3 Two- and three-dimensional visualization of $\gamma \mathrm{H} 2 \mathrm{AX}$ (green) and 53BP1 (red) DSB repair foci in spatially (3D) fixed NHDF-Neo fibroblasts irradiated with $1 \mathrm{~Gy}$ of accelerated ${ }^{11} \mathrm{~B}$ ions (LET $=138.1 \mathrm{keV} \mu \mathrm{m}^{-1}, E=8.1 \mathrm{MeV}$ per $\mathrm{n}$ ) in the perpendicular $\left(90^{\circ}\right)$ geometry. The cell shown here was fixed with paraformaldehyde $1 \mathrm{~h}$ after irradiation, followed by immunostaining for $\gamma \mathrm{H} 2 \mathrm{AX}$ and 53BP1 and counterstaining (chromatin) with DAPI (blue). The 2D maximum image (left) comprised 25 confocal slices (including slices above and below the cell nuclei) obtained with a $z$-step of $0.25 \mu \mathrm{m}$. A single 3D confocal slice (right) reveals the increased ability of this technique to distinguish $\gamma \mathrm{H} 2 \mathrm{AX} / 53 \mathrm{BP} 1$ foci in 3D space; an example of 3 foci that overlapped in the 2D image but were well separated in the 3D image is indicated by the red circle. Scale bars: $x-y, 5 \mu \mathrm{m}$; and $x-z$ and $y-z$, $2.5 \mu \mathrm{m}$. 


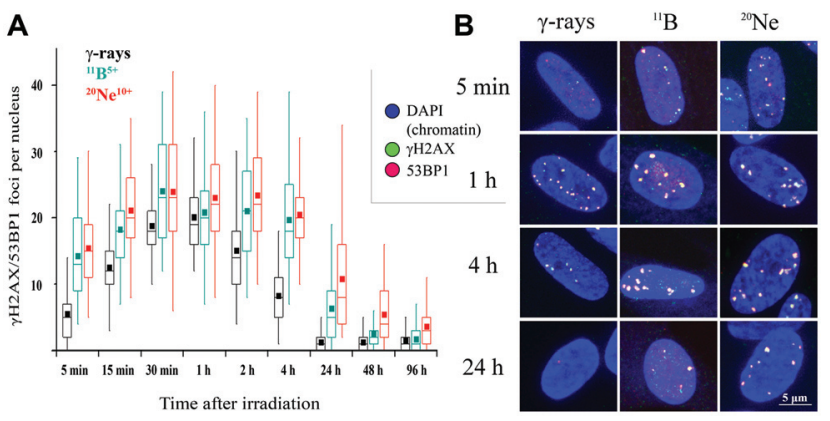

Fig. $5 \gamma \mathrm{H} 2 \mathrm{AX} / 53 \mathrm{BP} 1$ foci formation and loss (DSB repair dynamics) upon exposure to radiation of different qualities. NHDF-Neo cells were irradiated in the perpendicular $\left(90^{\circ}\right)$ geometry with 1 Gy of $\gamma$-rays, ${ }^{20} \mathrm{Ne}$

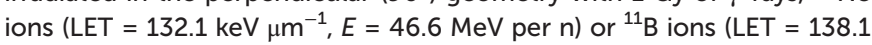
$\mathrm{keV} \mu \mathrm{m}^{-1}, E=8.1 \mathrm{MeV}$ per $\mathrm{n}$ ) and fixed at different times PI, as indicated. (A) Quantification of the number of $\gamma \mathrm{H} 2 \mathrm{AX} / 53 \mathrm{BP} 1$ foci in 3D images. Sham-irradiated cells contained (not shown) 0.1 foci per nucleus on average. The box-and-whisker plot indicates the mean (black square), median (median line inside the box), $25^{\text {th }}$ and $75^{\text {th }}$ percentiles (the top and bottom of box, respectively), and minimum and maximum (whiskers) of the pooled data from two experiments (approximately 100 counted cells). (B) Representative maximum intensity images of the corresponding cell nuclei. $\gamma \mathrm{H} 2 \mathrm{AX}$ (green), 53BP1 (red), chromatin (DAPI).

of $\gamma \mathrm{H} 2 \mathrm{AX} / 53 \mathrm{BP} 1$ foci upon irradiation was observed in cells irradiated with ${ }^{20} \mathrm{Ne}$, whereas the slowest production was observed in cells subjected to $\gamma$-rays. The opposite situation emerged for foci elimination. For cells exposed to both types of high-LET ions, the foci disappeared more slowly than in cells subjected to $\gamma$-rays. Four hours after irradiating the cells with ${ }^{20} \mathrm{Ne}$ or ${ }^{11} \mathrm{~B}$ ions, approximately $85 \%$ of the maximum numbers of $\gamma \mathrm{H} 2 \mathrm{AX} / 53 \mathrm{BP} 1$ foci persisted in the cells, whereas this fraction was only $41 \%$ for cells irradiated with $\gamma$-rays (Table 2). Moreover, although the $\gamma \mathrm{H} 2 \mathrm{AX} / 53 \mathrm{BP} 1$ foci per cell decreased to approximately 1 focus ( $6 \%$ of the maximum) at $24 \mathrm{~h}$ PI in $\gamma$-irradiated cells, approximately 6 (26\%) and 11 (45\%) foci remained in cells damaged by ${ }^{11} \mathrm{~B}$ and ${ }^{20} \mathrm{Ne}$ ions, respectively (Fig. 5 and Table 2). Consistent with this observation, preliminary data for cells exposed to another accelerated charged particle, ${ }^{12} \mathrm{C}\left(\mathrm{LET}=9.8 \mathrm{keV} \mu \mathrm{m}^{-1}, E=500 \mathrm{MeV}\right.$ per $\mathrm{n}, D=0.3 \mathrm{~Gy}$ ), revealed a similar delay in DSB repair; only a small number of foci disappeared from cells within the first

Table 2 Percentage of remaining $\gamma \mathrm{H} 2 \mathrm{AX} / 53 \mathrm{BP} 1$ foci after irradiation with the same dose of different radiation types

Time after irradiation

\begin{tabular}{lllrrr}
\cline { 2 - 5 } Radiation type & $2 \mathrm{~h}$ & $4 \mathrm{~h}$ & $24 \mathrm{~h}$ & $48 \mathrm{~h}$ & $96 \mathrm{~h}$ \\
\hline$\gamma$-Rays $\left({ }^{60} \mathrm{Co}\right)$ & 75.2 & 41.1 & 6.3 & 6.2 & 6.3 \\
${ }^{11} \mathrm{~B}$ & 87.7 & 84.7 & 26.4 & 10.6 & 7.1 \\
${ }^{20} \mathrm{Ne}$ & 97.9 & 85.7 & 45.1 & 22.8 & 15.1
\end{tabular}

Mean percent values of pooled data from two experiments (approximately 100 counted cells) are shown. The data were normalized to the peak numbers for each radiation type, i.e., the maximum number of foci scored for the particular radiation type, irrespective of the time PI.
$4 \mathrm{~h}$ PI, and $16 \%$ of these foci persisted in cells at $24 \mathrm{~h}$ PI (data not shown).

Interestingly, despite the similar LET values for ${ }^{11} \mathrm{~B}$ and ${ }^{20} \mathrm{Ne}$ ions and similar maximum numbers of $\gamma \mathrm{H} 2 \mathrm{AX} / 53 \mathrm{BP} 1$ foci induced by these radiation types, both the elimination kinetics of $\gamma \mathrm{H} 2 \mathrm{AX} / 53 \mathrm{BP} 1$ foci and their residual amounts measured at long periods of time (96 h) PI differed for these two radiation types. The number of $\gamma \mathrm{H} 2 \mathrm{AX} / 53 \mathrm{BP} 1$ foci persisting at this period of time in cells irradiated with boron ions (1.7 foci per nucleus) decreased to values typical for $\gamma$-irradiated cells (1.3 foci per nucleus), but approximately 4 foci per nucleus persisted in cells exposed to ${ }^{20} \mathrm{Ne}$ ions (Fig. 5). These values were significantly higher than shamirradiated (control) cells, with 0.1 foci per nucleus on average. These results demonstrate that the irradiation of NHDF cells with both ${ }^{20} \mathrm{Ne}$ and ${ }^{11} \mathrm{~B}$ high-LET ions (as well as ${ }^{12} \mathrm{C}$ ) delays the repair kinetics of DSBs compared with $\gamma$-rays, based on the persistence of $\gamma \mathrm{H} 2 \mathrm{AX} / 53 \mathrm{BP} 1$ foci in cells during PI periods. Although this finding was expected according to current theoretical knowledge (see Discussion), the surprising observation is that neon ions caused significantly more severe damage than boron ions with a very similar LET value. Therefore, we characterized the DNA damage resulting from boron and neon in more detail (next sections).

\section{2 $\gamma \mathrm{H} 2 \mathrm{AX} / 53 \mathrm{BP} 1$ focus streaks induced by high-LET ions with similar LET values and their changes with time post- irradiation}

In contrast to individual DSBs dispersed throughout the whole volumes of nuclei upon $\gamma$-ray irradiation, the exposure of cells to high-LET ions produced $\gamma \mathrm{H} 2 \mathrm{AX} / 53 \mathrm{BP} 1$ foci that were concentrated along the particle path ( $\gamma \mathrm{H} 2 \mathrm{AX} / 53 \mathrm{BP} 1$ focus streaks). We studied the morphology and dynamics of these streaks in cells irradiated at a sharp $\left(10^{\circ}\right)$ angle to the particle beam to maximize the resolution of separate foci using confocal microscopy. Doses of 1.0 and 1.2 Gy were used for ${ }^{11} \mathrm{~B}$ and ${ }^{20} \mathrm{Ne}$ ions, respectively, to ensure that an average of 3 particles traversed the nucleus in both cases. As early as $5 \mathrm{~min}$ PI, the streaks of $\gamma \mathrm{H} 2 \mathrm{AX}$ and 53BP1 foci were detectable in cells and were mutually colocalized. At least three $\gamma \mathrm{H} 2 \mathrm{AX} / 53 \mathrm{BP} 1$ foci were required to appear in a straight line parallel with the (known) direction of irradiating particles to be considered a streak. Streaks of both ions exhibited a grainy substructure and comprised closely spaced $\gamma \mathrm{H} 2 \mathrm{AX} / 53 \mathrm{BP} 1$ foci with nonstained "gaps" (Fig. 6). Within $1 \mathrm{~h}$ PI, streaks exhibited fully developed structures and the particle trajectories across the cell nuclei were clearly identified. As expected, most cells contained $3 \gamma \mathrm{H} 2 \mathrm{AX} / 53 \mathrm{BP} 1$ focus streaks.

Although the particles passed the nuclei along a linear path, some foci slightly deflected from this linear trajectory, particularly at longer periods of time PI ( $>2 \mathrm{~h}$ PI) (Fig. 6B). Beginning at $2 \mathrm{~h} \mathrm{PI}$, individual $\gamma \mathrm{H} 2 \mathrm{AX} / 53 \mathrm{BP} 1$ foci in the streaks also began to disappear, indicating the on-going repair of some DSBs. However, $22 \mathrm{~h}$ later ( $24 \mathrm{~h} \mathrm{PI}$ ), approximately $40 \%$ and $30 \%$ of the nuclei that had been irradiated with neon and boron ions still contained focus streaks, with typically one 


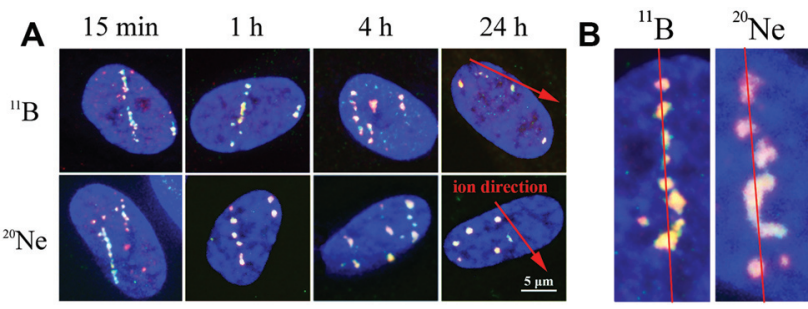

Fig. 6 Structures of $\gamma \mathrm{H} 2 \mathrm{AX} / 53 \mathrm{BP} 1$ focus streaks and their dynamic changes with time PI. NHDF-neo cell nuclei were exposed to an average of three ${ }^{20} \mathrm{Ne}$ or ${ }^{11} \mathrm{~B}$ ions (i.e., 1.2 and $1.0 \mathrm{~Gy}$, respectively) emitted at a sharp angle to the cell monolayer. Cells were spatially (3D) fixed at the indicated periods of time $\mathrm{Pl}$, and immunostaining for $\gamma \mathrm{H} 2 \mathrm{AX}$ (green) and 53BP1 (red) repair foci is presented. (A) Comparisons of $\gamma \mathrm{H} 2 \mathrm{AX} / 53 \mathrm{BP} 1$ focus streaks induced by boron and neon at the indicated periods $\mathrm{PI}$. (B) Detailed structures and deflections of foci from a linear particle track observed at $2 \mathrm{~h}$ after radiation exposure. Maximum images comprising $\sim 25$ superimposed $0.25 \mu \mathrm{m}$-thick confocal slices are shown in the $x-y$ plane in both A and B. Chromatin was counterstained with DAPI (blue).

streak in the nucleus (Fig. 6A). Within the next two days (96 h PI), all streaks had dissolved and only individual foci persisted in the nuclei. This observation highlights the successful repair of most DSBs and the existence of residual DNA damage. We also expect that seriously damaged cells died (see section 3.4), detached from the microscopic slides, and thus were undetectable in the confocal microscopy analyses.

\subsection{Morphology of $\gamma \mathrm{H} 2 \mathrm{AX} / 53 \mathrm{BP} 1$ foci as a function of radiation quality (evaluation of foci structure)}

The $\gamma \mathrm{H} 2 \mathrm{AX} / 53 \mathrm{BP} 1$ foci and focus streaks induced by high-LET boron and neon ions differed in morphology, specifically their sizes and shapes. Moreover, as described above, we recognized that some larger $\gamma \mathrm{H} 2 \mathrm{AX} / 53 \mathrm{BP} 1$ foci (later referred as to the $\gamma \mathrm{H} 2 \mathrm{AX} / 53 \mathrm{BP} 1$ clusters) comprised several smaller, closely spaced foci.

Therefore, we also analysed $\gamma \mathrm{H} 2 \mathrm{AX} / 53 \mathrm{BP} 1$ streaks in cells irradiated in the sharp-angle $\left(10^{\circ}\right)$ geometry prior to their spatial (3D) fixation in different periods of time PI to obtain more detailed insights (with better resolution power) into the morphology of $\gamma \mathrm{H} 2 \mathrm{AX} / 53 \mathrm{BP} 1$ foci and compare the structure and complexity of their clusters following exposure to the radiation types studied here. We focused on the following parameters: (a) the size of $\gamma \mathrm{H} 2 \mathrm{AX} / 53 \mathrm{BP} 1$ foci in the maximum images, (b) the circularity of $\gamma \mathrm{H} 2 \mathrm{AX} / 53 \mathrm{BP} 1$ foci in the maximum images $\left[4 \pi^{*}\left(\right.\right.$ area/perimeter $\left.\left.{ }^{2}\right)\right]$, and (c) the complexity of $\gamma \mathrm{H} 2 \mathrm{AX} / 53 \mathrm{BP} 1$ foci. All measurements were performed exactly as in the previous experiments with samples collected at $15 \mathrm{~min}$ and 1, 4, 24 and $96 \mathrm{~h}$ after irradiating cells with an average of 3 high-LET particles $\left(1 \mathrm{~Gy}{ }^{11} \mathrm{~B}\right.$ and 1.2 Gy of ${ }^{20} \mathrm{Ne}$ ions, respectively). Low-LET $\gamma$-rays administered at a dose of 1 Gy were used for comparison.

For all radiation types, the foci size rapidly increased beginning at $15 \mathrm{~min}$ PI, when the foci had not yet completely formed (Fig. 7A). The mean areas of $\gamma \mathrm{H} 2 \mathrm{AX} / 53 \mathrm{BP} 1$ foci induced by boron ions and $\gamma$-rays subsequently rapidly increased to maximum levels at $4 \mathrm{~h}$ PI. Afterwards, the mean areas of foci, which were larger for boron ions and smaller for $\gamma$-rays, plateaued at the maximum ( $\gamma$-rays) or slightly decreased (boron ions) by the end of the experiment (96 h PI). Interestingly, $\gamma \mathrm{H} 2 \mathrm{AX} / 53 \mathrm{BP} 1$ foci induced by neon ions behaved differently, increasing to values markedly exceeding the maximum sizes measured for $\gamma$-rays or boron ions until $24 \mathrm{~h}$ (Fig. 7A). Afterwards, the size of ${ }^{20} \mathrm{Ne}$-induced foci also decreased slightly, reaching values comparable to the values measured for boron ions or $\gamma$-rays at the end of the experiment (96 h PI). Despite the differences in the growth dynamics and final area of the foci induced by ${ }^{11} \mathrm{~B}$ and ${ }^{20} \mathrm{Ne}$, we concluded that both high-LET ions evoked (a) the formation of DSB foci on a much more rapid timescale than low-LET $\gamma$-rays, and (b) these foci were also larger.

The second measured parameter, the circularity of $\gamma \mathrm{H} 2 \mathrm{AX} /$ 53BP1 foci, also revealed significant quantitative differences between radiation types, reflecting their quality (Fig. 7B). Although the foci induced by $\gamma$-rays were mostly regular, with only small deviations from circularity, the foci showed marked
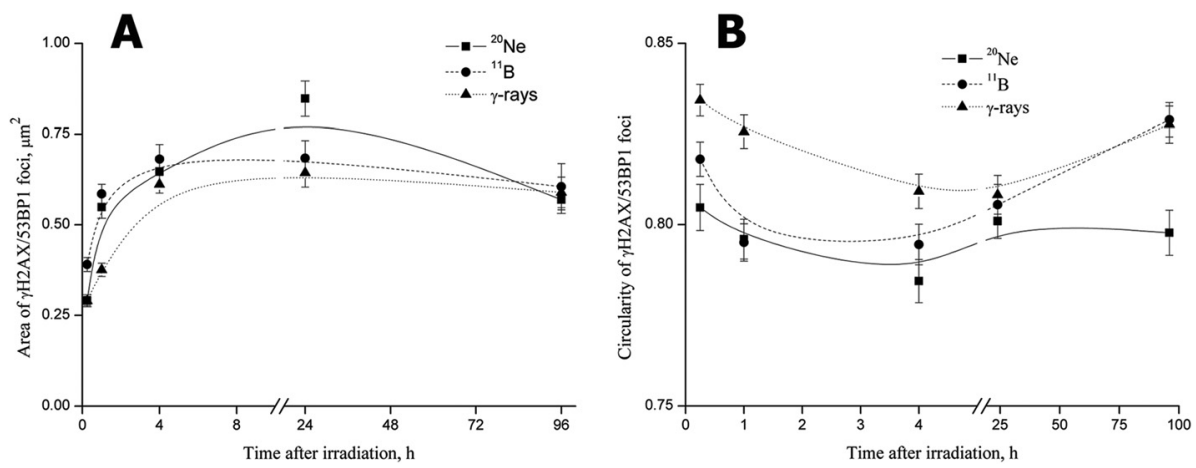

Fig. 7 Area (A) and circularity (B) of $\gamma \mathrm{H} 2 \mathrm{AX} / 53 \mathrm{BP} 1$ foci measured at various periods of time after the exposure of NHDF-Neo fibroblasts to $\gamma$-rays (1.0 Gy), boron ions (1.0 Gy) or neon ions (1.2 Gy). The slightly different doses applied for boron and neon ions ensured that an average of 3 particles traversed the nucleus in both cases. The measurements were performed in spatially (3D) fixed cells irradiated in the sharp-angle (10 ${ }^{\circ}$ geometry to maximize the resolution power. The data are presented as the means \pm standard errors calculated for pooled data from two experiments (>200 foci). B-spline curves were fitted using the least squares method in Origin 8 software. 
irregularities in shape after neon and boron irradiation, leading to lower circularity values. Interestingly, the circularity of $\gamma \mathrm{H} 2 \mathrm{AX} / 53 \mathrm{BP} 1$ foci changed over time PI, with the most prominent foci irregularity (deviation from the circularity) detected at $4 \mathrm{~h}$ PI for all radiation types. Specifically, we recognized three different profiles of changes in the circularity of the three radiation types studied (Fig. 7B). For $\gamma$-rays, the highest circularity was detected immediately after irradiation, after which it subsequently decreased and eventually returned to approximately the original values at the late (96 h PI) period PI. A similar profile was observed for neon ions, but both the initial (15 min PI) and the final (96 h PI) circularities were much lower than for $\gamma$-rays (and boron ions). Finally, for boron ions, the profile started with an intermediate initial circularity (lying between the values for neon ions and $\gamma$-rays) that decreased in the first hour PI, stagnated at lower values until $4 \mathrm{~h}$ PI, and subsequently started to increase, returning to the initial value at $24 \mathrm{~h}$ PI. However, in contrast to $\gamma$-rays and neon ions, the circularity of boron-induced foci increased compared with the initial value (measured upon irradiation) until it reached the value characteristic for $\gamma$-rays at $96 \mathrm{~h}$ PI.

The above-described analyses performed on the maximum images (2D superimpositions of individual confocal slices) clearly revealed the relationship between the radiation quality and morphology of $\gamma \mathrm{H} 2 \mathrm{AX} / 53 \mathrm{BP} 1$ foci. Therefore, in the next step, we thoroughly analysed the spatiotemporal changes in foci morphology in the 3D space using individual consecutive confocal slices $(0.25 \mu \mathrm{m}$-thick) from all 3D image stacks (25 slices per stack).

At all monitored periods of time PI (15 min to $96 \mathrm{~h}$ ), fractions of foci comprising several smaller foci appeared for all radiation types studied (representative images are shown in Fig. 8A). The yields and complexity of these clusters of foci largely depended on the radiation quality and the period of time PI (Fig. 8B). As expected, clusters of $\gamma \mathrm{H} 2 \mathrm{AX} / 53 \mathrm{BP} 1$ foci were rare upon exposure to low-LET $\gamma$-rays and these clusters exhibited low complexity: approximately $13 \%$ of foci appeared in clusters containing $2(11 \%)$ or occasionally 3 foci $(<2 \%)$. In contrast, one-half or almost three-quarters of all $\gamma \mathrm{H} 2 \mathrm{AX} / 53 \mathrm{BP} 1$ foci formed clusters in cells irradiated with boron and neon ions, respectively. In both cases, the complexity and fraction of focus clusters increased with time; both parameters were elevated until $4 \mathrm{~h}$ PI and subsequently decreased. Within $15 \mathrm{~min}$ PI, only $19 \%$ and $36 \%$ of foci occurred in clusters after boron and neon irradiation, respectively, whereas these fractions reached $58 \%$ and $71 \%$, respectively, at $4 \mathrm{~h}$ PI. At this time point, the focus clusters typically contained 2, 3 and 4 smaller foci; however, clusters comprising 5 to 8 foci were also detected, particularly in cells exposed to neon ions. Only insignificant changes in the focus cluster composition were observed between 4 and $24 \mathrm{~h}$ PI, followed by a marked reduction in both parameters, the foci complexity and proportion of focus clusters with a higher level of complexity, at 96 h PI. However, approximately 32\% (boron ions) and 44\% (neon ions) of foci were still located in clusters at that time point. In summary, neon ions induced the greatest incidence

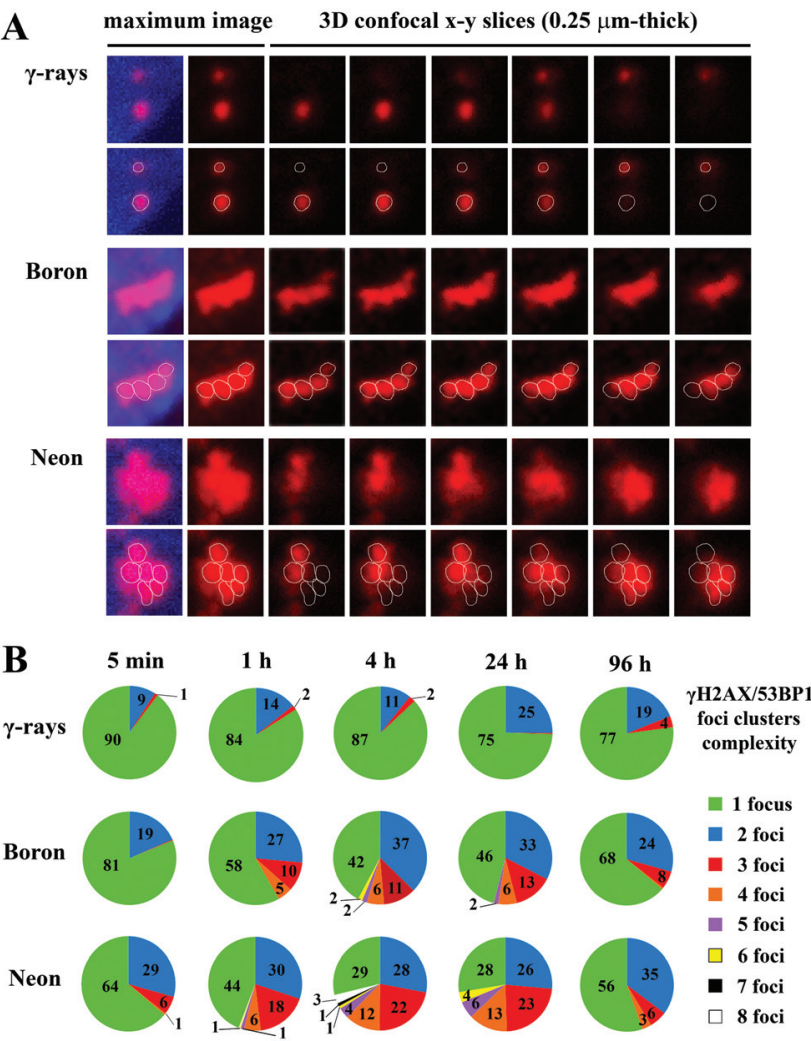

Fig. 8 Comparison of the complexity of $\gamma \mathrm{H} 2 \mathrm{AX} / 53 \mathrm{BP} 1$ focus clusters in spatially (3D) fixed NHDF-Neo fibroblasts irradiated with $\gamma$-rays, ${ }^{20} \mathrm{Ne}$ ions, or ${ }^{11} \mathrm{~B}$ ions. The dose was 1.0 Gy for $\gamma$-rays, 1.0 Gy for boron ions, and 1.2 Gy for neon ions to ensure that an average of 3 particles traversed the nucleus in both high-LET radiation cases. Cells were irradiated in the sharp-angle $\left(10^{\circ}\right)$ geometry. (A) Representative $\gamma \mathrm{H} 2 \mathrm{AX} /$ 53BP1 foci/focus clusters at the time period of their maximum complexity (4 h PI). The maximum (2D) images, shown on the left, comprise $\sim 25$ confocal slices obtained with a z-step of $0.25 \mu \mathrm{m}$. Individual consecutive confocal slices through the $\gamma \mathrm{H} 2 \mathrm{AX} / 53 \mathrm{BP} 1$ focus clusters (right columns) presented here subsequently show the composition of individual foci in the cluster and the ability to precisely describe their complexity. Only the 53BP1 signal is shown because this signal enables better discrimination of individual foci than the $\gamma \mathrm{H} 2 \mathrm{AX}$ signal. See Methods (chpt. 2.4) and Fig. 2 for details of the foci discrimination procedure. (B) The $\gamma \mathrm{H} 2 \mathrm{AX} / 53 \mathrm{BP} 1$ foci complexity was quantitatively compared for the radiation types studied. For different periods of time PI, the percentages of $\gamma \mathrm{H} 2 \mathrm{AX} / 53 \mathrm{BP} 1$ foci presented in clusters of a given complexity level are indicated. The mean values are calculated for pooled data from two experiments (>200 foci).

of the most complex clusters of foci. Compared with low-LET $\gamma$-rays and high-LET boron ions, a significantly greater proportion of $\gamma \mathrm{H} 2 \mathrm{AX} / 53 \mathrm{BP} 1$ focus clusters persisted in the nuclei of cells exposed to high-LET neon ions, even at $96 \mathrm{~h}$ after irradiation (Fig. 8).

\subsection{Apoptosis induction after irradiation with $\gamma$-rays and boron particles}

Based on the obtained results, the kinetics of $\gamma \mathrm{H} 2 \mathrm{AX} / 53 \mathrm{BP} 1$ foci elimination were markedly slower and the complexity of foci was greater in cells irradiated with high-LET radiation 
A

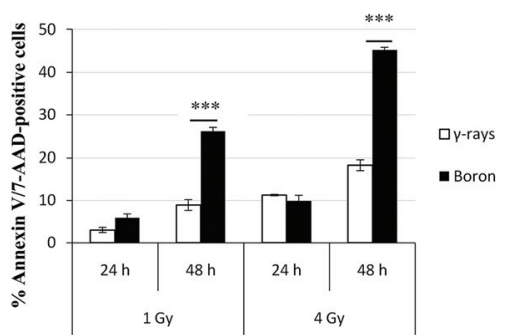

B

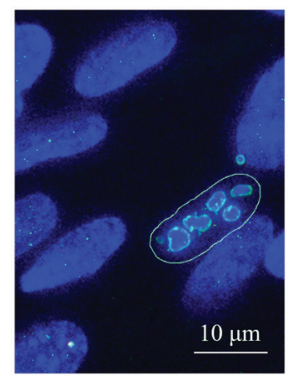

Fig. 9 Radiation-induced apoptosis of NHDF-Neo fibroblasts after exposure to $\gamma$-rays and ${ }^{11} \mathrm{~B}$ ions. (A) The induction of apoptosis was detected by flow cytometry (Muse ${ }^{\mathrm{TM}}$ Annexin-V \& Dead Cell Assay kit). Each bar represents the mean proportion of apoptotic cells after irradiation. The values were normalized to the "background" in the nonirradiated cells. $P$ values were calculated using the two-tailed $t$ test and

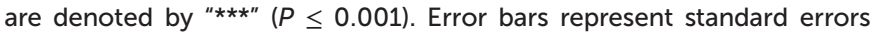
from three independent measurements of 5000 cells. (B) Representative image of a late apoptotic cell $(48 \mathrm{~h} \mathrm{PI})$, characterized by nuclear fragmentation and $\gamma \mathrm{H} 2 \mathrm{AX}$ (green) staining, after irradiation with $1 \mathrm{~Gy}$ of ${ }^{11} \mathrm{~B}$ ions. Chromatin was stained with DAPI (blue).

types than in cells irradiated with $\gamma$-rays. Hence, we simultaneously quantified Annexin V- and 7-amino-actinomycinpositive irradiated cells using flow cytometry to determine whether these findings were also correlated with increased cell death. Because of limited beam time availability, only cells irradiated with boron ions and $\gamma$-rays were studied. The results for the two radiation types applied in two doses (1 Gy and 4 Gy) and scored at two different periods of time (24 h and $48 \mathrm{~h}$ ) after irradiation are presented in Fig. 9. The values are expressed as the percentages of Annexin V-positive plus 7-AADpositive or negative cells normalized to the background level in non-irradiated cells. For both the 1 and 4 Gy doses, similar apoptosis rates occurred for $\gamma$-rays and boron ions at $24 \mathrm{~h} \mathrm{PI}$, regardless of the different LET values for these radiation types. This finding strikingly contrasts with the situation observed at $48 \mathrm{~h}$ PI, when boron ions induced approximately 3-fold higher numbers of positive cells than $\gamma$-rays. This difference was particularly prominent for the higher dose of 4 Gy. For both radiation types, the number of apoptotic cells clearly depended on the dose.

\section{Discussion}

To our knowledge, this study is the first to address the spatiotemporal aspects of DNA damage and repair after cells are exposed to different high-LET radiation types with similar lowenergy and LET values. Previous experimental research on the biological effects of high-LET radiation types have primarily focused on ions with high energies $(\geq 100 \mathrm{MeV}$ per $n)$, but results for low-energy $(<50 \mathrm{MeV}$ per $\mathrm{n})$ ions are still rare. Moreover, the mutually compared radiation types (in the literature) have typically largely differed in energy or LET values, frequently by orders of magnitude. ${ }^{6,30,62}$ Although this experimental design enables useful analyses of DNA damage (or other biological endpoints) related to LET or radiation energy, large differences in these parameters mask possible effects of other radiation qualities. The mechanism by which different high-LET particles affect the DNA molecule under conditions of similar LET and energy values thus remains poorly understood. We evaluated the extent, spatiotemporal behaviour and repair of DSB damage in normal human neonatal dermal fibroblasts irradiated with either boron or neon ions that had been accelerated to similar lower energies and similar LET values (see Table 1 ) or $\gamma$-rays, a representative low-LET radiation, to address this current gap in knowledge.

For many biological endpoints, the RBE increases with LET. However, for different radiation types, LET values oscillating between 100 and $200 \mathrm{keV} \mu \mathrm{m}^{-1}$ may correspond to the maximum RBE, depending on the physical parameters of the particle, cell type and biological endpoint studied. ${ }^{20-22,63}$ Alternatively, as shown in the present study, radiation types with similar LET values produce DNA damage with different complexities and spatiotemporal behaviours. Because increased DNA damage complexity was found to be correlated with slower DSB repair and increased cell death, our results confirm the hypothesis that the spatiotemporal characteristics of energy deposition and DNA damage, which cannot be simply described by LET, play a crucial role in determining the RBE of different radiation types.

Hence, micro-morphological analyses of DNA damage, as performed in the present study, significantly contribute to the current understanding of the mechanisms by which low-LET and high-LET radiation types kill cells and consequently the mystery of radiation RBE. To date, the knowledge in these areas is incomplete and optical microscopy approaches enabling detailed analyses of DSB damage under physiological conditions remain challenging. ${ }^{64}$ For example, recent works attempting to study DSB damage in cells exposed $\left(90^{\circ}\right.$ geome-

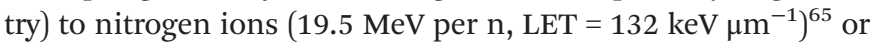
$\alpha$ particles $\left(0.75 \mathrm{MeV} \text { per } \mathrm{n}, \mathrm{LET}=125 \mathrm{keV} \mu \mathrm{m}^{-1}\right)^{66}$ using classical confocal microscopy failed to distinguish a substructure of $\gamma \mathrm{H} 2 \mathrm{AX}$ focus clusters, although they analysed cell nuclei in the 3D space. Therefore, various super-resolution 'nanoscopy' methods that increase the resolution power of optical microscopy up to several nanometres have high expectations. $^{36,64,67}$ Unfortunately, studies taking advantage of optical super-resolution techniques remain rare and are extremely technically demanding (depending on the method); hence, studies have typically been limited to analyses of relatively small datasets. ${ }^{35,61,68}$ Electron microscopy offers a superior resolution power that enables the precise quantification of DSB complexity; ${ }^{38-40,69,70}$ however, the necessity of sample sectioning makes analyses of entire particle tracks in space and time difficult. Moreover, experimental artefacts might appear, reflecting (harmful) sample fixation steps, such as dehydration or freezing (reviewed by Winey et al. ${ }^{71}$ ).

Therefore, in the present study, we used dual-immunofluorescence high-resolution confocal microscopy on spatially (3D) fixed cells irradiated at a sharp angle. ${ }^{59}$ Based on this approach, we successfully visualized a detailed substructure of 
colocalized $\gamma \mathrm{H} 2 \mathrm{AX}$ and 53BP1 focus streaks along ${ }^{11} \mathrm{~B}$ and ${ }^{20} \mathrm{Ne}$ particle tracks in situ and in large datasets. Selective scoring of colocalized $\gamma \mathrm{H} 2 \mathrm{AX}$ and 53BP1 foci improved the precision of these analyses and increased the probability of observing unrepaired DSBs, especially in later periods of time PI (see Methods, chapter 2.4 for explanation). The exclusion of cells with non-colocalizing signals (rare cases) also reduced the numbers of inefficiently immunostained cells and non-G1cells $^{52-54}$ included in the analyses (we studied non-synchronized cell populations to better reflect the situation in irradiated tissues; the cultures contained $>80 \%$ G1 cells at the time of irradiation).

In contrast with the results reported by Costes et $a .^{65}$ or Antonelli et al., ${ }^{66} 3 \mathrm{D}$ analyses of confocal image stacks performed in this study improved the detection of $\gamma \mathrm{H} 2 \mathrm{AX} / 53 \mathrm{BP} 1$ (sub)foci by approximately $40 \%$ compared with a simple $2 \mathrm{D}$ analysis (see Fig. 4). Nevertheless, some potential and real limitations of the present study should be considered. First, cell fixation may influence chromatin structure. Therefore, we confronted the morphology of the cell nuclei (thickness and the maximal length $v s$. thickness ratio) of the fibroblasts (as a widely accepted model for exploring normal tissue radiosensitivity) ${ }^{60}$ after $3 \mathrm{D}$ fixation with the results of other studies. $^{61,72,73}$ The measurements (nucleus thickness of approximately $2.7-3.4 \mu \mathrm{m}$ ) confirmed that the complexity of $\gamma \mathrm{H} 2 \mathrm{AX} / 53 \mathrm{BP} 1$ focus clusters predominantly reflects the deposition of radiation energy, without a significant contribution of cell flattening. Indeed, closer approach of very tightly spaced $\gamma \mathrm{H} 2 \mathrm{AX} / 53 \mathrm{BP} 1$ foci in the focus streaks of high-LET ions, potentially provoked by fixation-mediated cell flattening, would lead to overlapping foci and underestimation of focus cluster complexity, rather than to a dramatic increase in this parameter (e.g., because of fusions between foci located inside of and more distant from the main particle track). In any case, relative comparisons should be unaffected, as the same potential effect was detected for all cells.

Second, as previously reported by Jakob et al. ${ }^{29}$ sharp-angle cell irradiation allowed us to substantially increase the resolution power; this strategy reduced limitations arising from fibroblast flatness and the insufficient resolution power of confocal microscopy in the vertical $(z)$-direction. ${ }^{74}$ To identify individual foci within clusters, we combined information on cluster morphology, fluorescence heat maps, and fluorescence intensity profiles along the paths intersecting putative foci in non-overexposed parts of the cluster (see Methods, Fig. 2). Although high precision of the spatiotemporal analysis was achieved, serious technical restrictions (low resolution power of confocal microscopy, leading to a certain amount of artificial overlap in complex arrangements of foci due to diffraction; reviewed in Waters $^{74}$ ) as well as biological reality (spreading of H2AX phosphorylation over 1-2 Mb chromatin domains and overlapping signals) prevented the discrimination of individual DSBs in multiple lesions ( $\geq 2$ DSBs within 1-2 helical turns). Hence, the precise boundaries and, thus, the numbers of very closely spaced DSB foci were only estimated. The biological nature of the complex $\gamma \mathrm{H} 2 \mathrm{AX} / 53 \mathrm{BP} 1$ focus clusters observed in the present study and the precise numbers of DSBs present in $\gamma \mathrm{H} 2 \mathrm{AX} / 53 \mathrm{BP} 1$ foci/focus clusters (in general) therefore remain uncertain and must be further studied. Several recent reports $^{75-78}$ have suggested that super-resolution localization light microscopy offers an exciting but still challenging means of analysing the same biological specimens in parallel with confocal microscopy, but with much more detailed data. This strategy could be used to obtain answers to many questions discussed in this chapter in the near future.

Taking advantage of current approach, the microdosimetric complexity of $\gamma \mathrm{H} 2 \mathrm{AX} / 53 \mathrm{BP} 1$ (DSB) repair foci differs not only for cells irradiated with low-LET $\gamma$-rays and high-LET radiation types, as expected, but also for cells irradiated with boron ions and neon ions, which have very similar LET and low-energy values. Mathematical modelling/simulations and novel ultrasensitive immunogold-labelling transmission electron microscopy (TEM) provides clear evidence that high-LET radiation causes complex DSBs, and in contrast to low-LET radiation, the majority of $\gamma \mathrm{H} 2 \mathrm{AX}$ foci contain more than one DSB. ${ }^{32,38,39}$ As recently reviewed by Goodhead et al. (2015), approximately 20\% ( $\gamma$-rays) and 70\% (high-LET ions) of DSB damage sites contain at least three DNA-chain breaks, according to simulations. ${ }^{7}$ Based on our direct measurements $(1 \mathrm{~h}$ PI), approximately $16 \%, 42 \%$, and $56 \%$ of $\gamma \mathrm{H} 2 \mathrm{AX} / 53 \mathrm{BP} 1$ foci generated from $\gamma$-rays, boron ions, and neon ions, respectively, exist in clusters with additional foci. Nevertheless, these numbers were not able to be directly compared with other studies that typically quantify the proportion of simple foci to DSB clusters. In any case, consistent with previous findings, ${ }^{38,40,61}$ our 3D analysis revealed highly clustered (>3) $\gamma \mathrm{H} 2 \mathrm{AX} / 53 \mathrm{BP} 1$ foci only after exposure to high-LET particles, whereas clusters observed after $\gamma$-irradiation comprised a maximum of two or occasionally (2\%) three foci.

The difference between DSB complexity following the exposure of cells to $\gamma$-rays and high-LET ions (boron or neon) was primarily explained by the different characters of energy deposition (ionization density and distribution) by these radiation types. However, based on the present and previous results, ${ }^{24,67,79}$ we propose that not only the character of energy deposition per se but also the character of energy deposition in the context of higher-order chromatin structure ${ }^{80}$ (see Fig. 10F) significantly contributes to the increased complexity of DNA damage and RBE following exposure to high-LET radiation types. Specifically, high-LET radiation types attack condensed (hetero)chromatin, which contains a much higher density of potential DNA targets than decondensed (eu)chromatin, more efficiently than low-LET radiation types ${ }^{79}$ (reviewed in ref. 24 and 67). However, $\gamma$-rays preferentially damage low-density chromatin, since, in condensed (hetero)chromatin, more abundant (hetero)chromatin-binding proteins better shield the DNA from free radicals largely mediating the harmful effects of low-LET radiation. Therefore, it is important not only how, but also where the radiation energy is released in the cell nucleus (also see Fig. 10F).

The complexity of DSBs was inversely correlated with the disappearance of $\gamma \mathrm{H} 2 \mathrm{AX} / 53 \mathrm{BP} 1$ foci from the cell nuclei, i.e., 

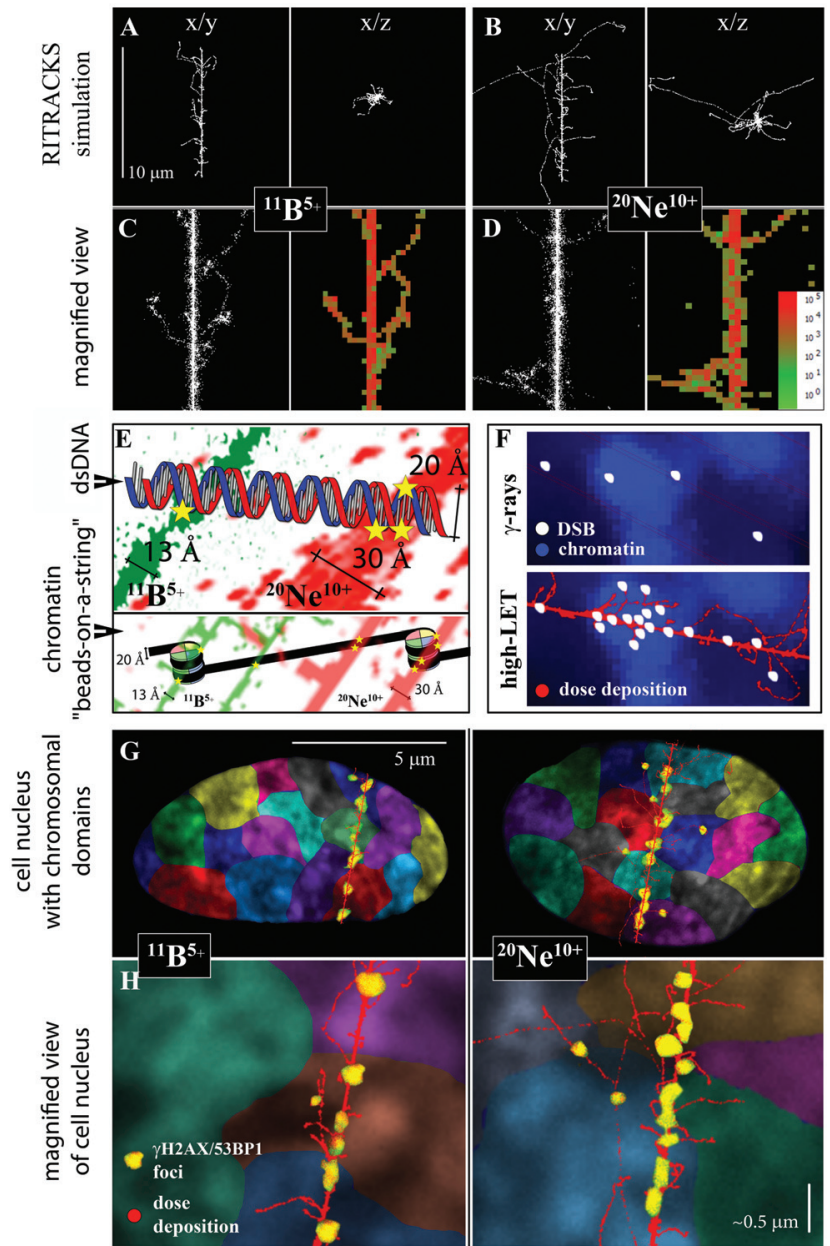

Fig. 10 Relationships between radiation energy deposition, higher-order chromatin structure and DNA double-strand break induction for $\gamma$-rays and ${ }^{11} \mathrm{~B}$ and ${ }^{20} \mathrm{Ne}$ ions (see Table 1 for radiation parameters). A, B. Simulations of $10 \mu \mathrm{m}$-long track structures for ${ }^{11} \mathrm{~B}$ and ${ }^{20} \mathrm{Ne}$ ions using RITRACS software; the range and distribution of $\delta$-electrons relative to the track core can be observed in $x / y$ and $x / z$ projections. C, D. Detailed views along the tracks, with emphasis on the core structure. Free radical tracks (left panel) are shown together with dose deposition tracks (right panels). Dose deposition decreases from red to green, as indicated. The simulations show that $\delta$-electrons penetrate deeper with neon ions, while they are more concentrated along the track core with boron ions. Nevertheless, the combined action of the neon particle itself plus low-energy electrons (that do not escape from the track core) generates a thick track core, approximately twice as thick as the boron ion. E. Compared with ${ }^{11} \mathrm{~B}$ ions, a wider track core of ${ }^{20} \mathrm{Ne}$ ions (which is still highly concentrated compared, for example, to particles of high energy) directly damages both strands of the DNA molecule with a higher probability/frequency. Moreover, ${ }^{20} \mathrm{Ne}$ ions can more easily attack both DNA turns around the nucleosome at the same time (bottom image). Chromatin is shown at the organization level of "beads-on-a-string" for simplification. F. Relationships between dose deposition, higher-order chromatin structure and DSB induction. $\gamma$-rays (top image) induce DSBs randomly across the cell nucleus, with euchromatin being more sensitive than heterochromatin to free radicals (which largely mediate harmful effects of low-LET radiation), since heterochromatin is better protected by larger amounts of (hetero)chromatin-binding proteins. ${ }^{24,64,79}$ In contrast, high-LET radiation (bottom image) deposits energy in a concentrated manner along the particle track, which causes DNA damage that cannot be prevented by the chromatin structure. Condensed (hetero) chromatin provides more DNA targets per volume unit and is therefore more seriously damaged (fragmented) by high-LET particles. ${ }^{64} \mathrm{G}$, $\mathrm{H}$. Relationships between the character of energy deposition, higher-order chromatin structure, and induction of chromosomal aberrations. The dose deposition (red) simulated for ${ }^{11} \mathrm{~B}$ and ${ }^{20} \mathrm{Ne}$ ions by the RITRACKS code is overlaid onto the cell nucleus, with schematically illustrated chromosomal territories (various colours) and $\gamma \mathrm{H} 2 \mathrm{AX} / 53 \mathrm{BP} 1$ foci (yellow). A slightly greater complexity of $\gamma \mathrm{H} 2 \mathrm{AX} / 53 \mathrm{BP} 1$ foci in cells irradiated with ${ }^{20} \mathrm{Ne}$ ions (compared with ${ }^{11} \mathrm{~B}$ ions) is indicated by their larger size. The higher-order chromatin structure is shown via DAPI staining (condensed chromatin is intensively stained). Both ions and kick-off electrons of low energy generate extensive damage along the particle path and thus complex chromosomal aberrations. In addition, ${ }^{20} \mathrm{Ne}$ ions emit longer-range $\delta$-electrons than ${ }^{11} \mathrm{~B}$ ions (panel A vs. B). These electrons radiate to sufficient distances from the track core to cause DSBs in the neighbouring chromosomal territories; nevertheless, these DSBs may still be sufficiently close to interact with DSBs along the particle core, particularly if we consider the chromatin fragmentation and decondensation caused by irradiation and the associated, though limited, chromatin movement. Typically, damaged chromatin (labelled with 53BP1-GFP in living cells) is displaced at a distance of approximately $1 \mu \mathrm{m}$ in cells irradiated with accelerated ions, but the displacement of $<2 \%$ foci occasionally exceeds $5 \mu \mathrm{m}$ in the $12 \mathrm{~h}$ interval after $\mathrm{Ni}$ irradiation. ${ }^{93}$ Therefore, we propose that chromosomal aberrations formed with ${ }^{20} \mathrm{Ne}$ ions may involve more chromosomes than those that appear with ${ }^{11} \mathrm{~B}$ ions, and their complexity in terms of the number of DNA breaks can be higher, as shown in the present study. Consider that human skin fibroblasts are flat cells (as shown on the image), and even more chromosomal territories may participate in chromosomal aberrations in spherical cells. ${ }^{17,94,95}$ Panel $\mathrm{H}$ : Section of panel G magnified approximately 10 times. Scale bar, approximately $0.5 \mu \mathrm{m}$ (schematic illustration). 
the kinetics and efficiency of DSB repair, and cell survival. Consistent with some earlier reports, ${ }^{31,61,64,66,79-81}$ simple (and on principle frequently euchromatic ${ }^{79}$ ) $\gamma \mathrm{H} 2 \mathrm{AX} / 53 \mathrm{BP} 1$ foci generated by $\gamma$-rays were largely eliminated within the first $(4 \mathrm{~h})$ hours PI in the present study. Similar findings of the rapid repair and disappearance of non-clustered (smaller) individual $\gamma \mathrm{H} 2 \mathrm{AX} / 53 \mathrm{BP} 1$ foci were observed in cells irradiated with high-LET ions $\left({ }^{11} \mathrm{~B}\right.$ and $\left.{ }^{20} \mathrm{Ne}\right)$. However, large $\gamma \mathrm{H} 2 \mathrm{AX} / 53 \mathrm{BP} 1$ foci, and particularly clusters of these foci, were repaired with difficulty irrespective of their origin in euchromatin or heterochromatin, and they persisted in the cell nuclei for much longer periods of 1 day $\left({ }^{11} \mathrm{~B}\right)$ or more $\left({ }^{20} \mathrm{Ne}\right)$ after irradiation. For ${ }^{20} \mathrm{Ne}$ ions that produced the most complex DSB lesions in this study, approximately $3 / 4$ of DSB foci persisting at $24 \mathrm{~h}$ PI formed clusters. These results precisely correspond to an interesting study by Jakob et al. ${ }^{48}$ who observed the slower repair of DSBs generated by carbon ions than of DSBs produced by $\gamma$-rays, and this delayed repair was even higher for heterochromatin-associated damage. Based on the evidence obtained in this and previous studies, we conclude that both DSB complexity and the chromatin environment surrounding DSBs significantly influence the repair of initially formed lesions (appearing as a consequence of energy deposition), which at least partially explains the higher RBE of high-LET ions.

The average complexity of clusters and number of clusters per cell gradually increased with time PI and started to decrease only after $24 \mathrm{~h}$ PI. This scenario suggests the gradual processing of less complex and individual DSBs within the clusters $^{61}$ and highlights the long-term persistence of complex DSB clusters. An alternative, but not mutually exclusive possibility is that these repair kinetics may reflect the formation of DSB focus clusters over a longer time post-irradiation, due to chromatin movement provoked by DSB repair processes, as described below. The cells with unrepeatable DSB clusters (of either origin) are expected to die or undergo mutagenesis at later periods of time PI. Indeed, a much higher proportion of cells underwent apoptosis following irradiation with boron ions (almost $50 \%$ of cells exposed to a 4 Gy dose) compared with $\gamma$-rays (less than $20 \%$ of cells exposed to a 4 Gy dose), indicating that (a) DSBs produced by high-LET ions are repaired but only with difficulty and (b) the proportions of cells with complex $\gamma \mathrm{H} 2 \mathrm{AX} / 53 \mathrm{BP} 1$ foci and the complexity of persistent DSBs at $>24 \mathrm{~h}$ PI may be even greater than reported here (because the seriously damaged cells died, detached from the microscopic slides and escaped detection).

Moreover, concerning the long period of time examined post-irradiation (up to $96 \mathrm{~h} \mathrm{PI}$ ), a proportion of cells may also die due to mitotic catastrophe, since as was only recently shown, ${ }^{61,82}$ even cells with relatively high numbers (e.g., 10-15) of DSBs can enter mitosis under some circumstances. These conclusions are consistent with the combined results of previous studies of carbon and ferrous ions. ${ }^{38,39,61}$

Finally, we cannot exclude the possibility that previously repaired cells may overpopulate cells still harbouring damage and, thus, further decrease the average number of foci per nucleus observed in later time periods PI. Since the critical level of DSBs $(<10-20)^{82}$ allowing cells to enter mitosis is reached more rapidly (within approximately $4 \mathrm{~h}$ PI) using $\gamma$-rays than using both high-LET ions ( $>24 \mathrm{~h} \mathrm{PI}$ ), this effect may eventually lead to underestimation of DSBs, especially in $\gamma$-irradiated cells. However, any significant effect of cell division on DSB repair kinetics and differences in such effects between the different types of radiation studied are unlikely since (a) only a small fraction of fibroblasts were dividing; (b) only background DSB numbers were detected in $\gamma$-irradiated cells at $24 \mathrm{~h}$ PI (and this number plateaued at later times); and (c) the most prominent differences in DSB repair kinetics were observed at PI times $<4 \mathrm{~h}$. We also calculated the average numbers of $\gamma \mathrm{H} 2 \mathrm{AX} / 53 \mathrm{BP} 1$ foci per nucleus specifically in $\gamma \mathrm{H} 2 \mathrm{AX} / 53 \mathrm{BP} 1-$ positive cells (not shown), which resulted in the same trends/conclusions obtained from analyses performed for whole cell populations $(\gamma \mathrm{H} 2 \mathrm{AX}$-positive $+\gamma \mathrm{H} 2 \mathrm{AX}$-negative cells). Moreover, most cells exposed to $\gamma$-rays contained $<20$ DSB (mean $=$ approximately 10 DSBs) foci per nucleus at $4 \mathrm{~h}$ PI, and damaged cells could therefore theoretically also undergo mitosis, similar to undamaged cells.

An additional level of DSB complexity that negatively influences DSB repair may paradoxically appear as a side effect of repair processes per se. First, the processing of complex (even non-DSB) lesions directly generates secondary DSBs. ${ }^{83,84}$ Second, the heterochromatin structure has consistently been reported to represent a barrier to DNA repair and thus DSBs in condensed heterochromatin are repaired but only with difficulty, with slower kinetics and lower efficiency than DSBs in euchromatin. ${ }^{38-40,48,79,85}$ The repair of heterochromatic DSBs requires extensive (hetero)chromatin decondensation, which in turn locally mobilizes damaged chromatin domains to some extent. ${ }^{25,81,86,87}$ This increased mobility of heterochromatic DSBs can result in collisions of two or more DSBs and the formation of secondary DSB clusters (the term 'secondary' distinguishes these clusters from the 'primary' clusters discussed above, which are directly formed by high-LET particles due to localized energy deposition). Although this phenomenon occurs occasionally upon irradiation with $\gamma$-rays, its importance has dramatically increased for cells exposed to high-LET radiation types, in which the local chromatin structure is highly fragmented and DSBs are concentrated along a relatively thin particle path, i.e., located in close proximity to one another. Indeed, the secondary DSB clusters observed after high-LET irradiation are in fact frequently higher order clusters comprising primary DSB clusters. ${ }^{67,85}$

Consistent with this scenario, we observed deflections of some foci from otherwise linear particle tracks and these 'mobile' foci mostly occurred at the border between condensed (hetero)chromatin and decondensed (eu)chromatin domains. The protrusion of heterochromatic DSBs into decondensed (eu)chromatin has also been reported in other studies upon high-LET ${ }^{26,48}$ and low-LET ${ }^{81,86,88}$ irradiation (reviewed by Falk et al. $;^{24}$ Chiolo et al. ${ }^{89}$ ). Moreover, the highest numbers of clusters were detected between 4 and $24 \mathrm{~h}$, i.e., when the DSB repair of non-complex lesions is mostly complete. During this PI period, the clusters also showed the greatest irregularity and 
complexity, with 2 to 5 smaller foci per cluster observed in boron-irradiated cells and up to 8 foci observed in neon-irradiated cells. Together, these results highlight the problematic spatiotemporal stabilization of complex $\gamma \mathrm{H} 2 \mathrm{AX} / 53 \mathrm{BP} 1$ focus clusters, particularly in the initial phase and most active period of DSB repair. Conceivably, after high-LET irradiation, simultaneous chromatin fragmentation and dispersion supported by chromatin decondensation occurring in the frame of repair processes locally mobilize chromatin to a much greater extent than in cells exposed to low-LET $\gamma$-rays. This phenomenon dramatically increases the complexity of DSB lesions and the risk of repair errors or failure. Finally, these observations demonstrate that two phenomena - energy deposition, leading to primary chromatin fragmentation, and DNA repair, provoking secondary chromatin decondensation - contribute to (restricted) chromatin movement and formation of (complex/ multiple) DSB clusters and chromosomal aberrations in cells exposed to high-LET ions.

The greater complexity and delayed repair of DSB lesions induced by neon ions compared with that induced by boron ions, which was observed in our morphological and kinetic studies, is harder to explain than the differences between high-LET and low-LET types of radiation. For low radiation energies, the $\delta$-electrons emitted by the transversing particle are expected to significantly damage DNA inside or close to the track core since they do not have sufficient energy to escape. Consistently, for both boron and neon ions, we observed only occasional $\gamma \mathrm{H} 2 \mathrm{AX} / 53 \mathrm{BP} 1$ foci (more frequent for neon ions) that were attributed to the activity of $\delta$-electrons at sites located outside of $\gamma \mathrm{H} 2 \mathrm{AX} / 53 \mathrm{BP} 1$ focus streaks. In the proximity of the track core, some large $\gamma \mathrm{H} 2 \mathrm{AX} / 53 \mathrm{BP} 1$ foci diverged from the linearity of the streaks, in addition to small $\gamma \mathrm{H} 2 \mathrm{AX} / 53 \mathrm{BP} 1$ foci produced by $\delta$-electrons (see Fig. 6); these objects likely correspond to complex foci relocated from the track core or increased energy deposition at the ends of $\delta$-electron tracks. ${ }^{90-92}$ Whether $\delta$-electron ends can generate DSBs with a sufficient density to form multiple DSBs is unclear; ${ }^{90-92}$ in any case, such DSB clusters would be less complex than clusters inside the track core. Hence, based on the relatively large size of observed out-of-line foci and the gradually decreasing linearity of the particle tracks with the PI period, we consider the first scenario to be more likely/important. The track core therefore probably plays a crucial role in influencing the characteristics of DSB damage under the conditions of the present study, although long-range $\delta$-electrons may also influence characteristics such as the complexity of chromosomal aberrations (see Fig. 10).

Therefore, we complemented our experiments with theoretical calculations on the track core (as described by Chatterjee, $1993,{ }^{57}$ see the Methods) to determine potential factors that may explain why neon ions induce DNA damage of greater complexity than boron ions. The results revealed a core radius of only $13 \AA$ for boron ions but $30 \AA$ for neon ions. An approximately two-times wider track "core" for ${ }^{20} \mathrm{Ne}$ ions was also observed in RITRACKS simulations (Fig. 10). Based on these results, we propose that neon ions generate a wider particle track core that is better able to cause complex DNA (DSB) lesions than the thinner track core of boron ions. This conclusion is not in disagreement with the findings of Saha $e t$ al. $(2014),{ }^{30}$ who associated a thinner track of low-energy ions (more concentrated energy deposition) with more efficient DNA damage induction than was associated with a wider track of high-energy ions - the tracks of the ${ }^{11} \mathrm{~B}$ and ${ }^{20} \mathrm{Ne}$ ions used here are both much thinner than the tracks of high-energy ions.

The proposed relationships between the particle track structures of ${ }^{11} \mathrm{~B}$ and ${ }^{20} \mathrm{Ne}$ ions, the higher-order chromatin architecture and the formation of (complex) DSBs and chromosomal aberrations (discussed here as the endpoint of irradiation most directly related to DSB induction) are illustrated in Fig. 10. Briefly, both high-LET ions $\left({ }^{11} \mathrm{~B}\right.$ and $\left.{ }^{20} \mathrm{Ne}\right)$ and kick-off electrons of low energy generate extensive damage along the particle track core, which leads to complex chromosomal aberrations that may involve chromosomes whose (interphase) territories are intersected by the particle. A wider but still highly focused (dense energy deposition) track core of ${ }^{20} \mathrm{Ne}$ ions can more efficiently induce DSBs than too narrow track core of ${ }^{11} \mathrm{~B}$ ions. In addition, ${ }^{20} \mathrm{Ne}$ ions emit longer-range $\delta$-electrons than ${ }^{11} \mathrm{~B}$ ions. These electrons radiate to sufficient distances from the track core and may cause numerous DSBs, mostly at the end of their tracks, i.e., in neighbouring (more distant) chromosomal territories. Nevertheless, these DSBs may be still sufficiently close to interact with DSBs along the particle core, particularly if we consider the chromatin fragmentation and decondensation caused by irradiation and the associated, though limited, chromatin movement. Typically, damaged chromatin (labelled with 53BP1-GFP in living cells) may be displaced by a distance of approximately $1 \mu \mathrm{m}$ in cells irradiated with accelerated ions, although relocation occasionally $(<2 \%$ foci) exceeds $5 \mu \mathrm{m}$ during the $12 \mathrm{~h}$ interval after high-LET nickel ion exposure. ${ }^{93}$ Therefore, we propose that chromosomal aberrations formed with ${ }^{20} \mathrm{Ne}$ ions may involve more chromosomes than appear in association with ${ }^{11} \mathrm{~B}$ ions, and their complexity in terms of the number of DNA breaks can be higher, as shown in the present study (Fig. 10G and H; note that more chromosomal territories may participate in chromosomal aberrations in spherical cells than in flat fibroblasts). ${ }^{17,94,95}$ Nevertheless, this hypothesis remains to be experimentally confirmed.

Moreover, the radiation types used in the present study differed in particle charge $\left({ }^{20} \mathrm{Ne}=10+,{ }^{11} \mathrm{~B}=5+\right)$, which was not considered in our calculations. Because the higher charge of Ne ions is reasonably expected to influence the microdosimetric distribution of ionization and thus significantly contribute to the track (core) structure, ${ }^{96,97}$ we can hypothesize that it also increases DNA damage complexity under conditions of similar LET and energy values for different radiation types. However, the physical quality of the particle, which primarily determines the characteristics of DNA damage under conditions of similar LET and energy values for the radiation types, should be further investigated.

The $\gamma \mathrm{H} 2 \mathrm{AX} / 53 \mathrm{BP} 1$ foci/focus clusters generated by boron and neon ions and $\gamma$-rays differed not only in complexity but 
also in structural parameters and spatiotemporal behaviours. Neon ions provoked the most rapid formation of $\gamma \mathrm{H} 2 \mathrm{AX} / 53 \mathrm{BP} 1$ foci in irradiated cells and neon ions also induced the largest and most irregular foci (the lowest circularity). The results for boron ions were generally more similar to neon ions than to $\gamma$-rays. Faster formation of DSB foci in cells irradiated with high-LET ions compared with low-LET $\gamma$-rays is not unprecedented in radiobiology, ${ }^{34,93}$ but negative observations ${ }^{65,98}$ leave the question open. We postulate that chromatin fragmentation by high-LET radiation types opens damaged chromatin sites for DSB sensors and repair factors, which is manifested in rapid focus formation, occurring faster and to greater extent than fragmentation induced by $\gamma$-rays. Alternatively, but not mutually exclusively, clustered DSBs (together with factors such as high local concentrations of radicals), which represent a serious threat to genome integrity, may induce a more severe shock to the cells than a comparable, or even higher, level of spatially separated DSBs, and the repair systems are therefore activated faster or at a higher level. Finally, reflecting the character of energy deposition (highly localized $v s$. dispersed, with high-LET and low-LET radiation, respectively), primary DSB clusters generated by high-LET ions appear in cells immediately following irradiation, whereas the formation of occasional secondary clusters induced by $\gamma$-rays requires additional time. As previously discussed, primary clusters originate from physico-chemical processes occurring immediately after irradiation, while secondary clusters form later, as a consequence of restricted chromatin movement provoked by DNA repair; ${ }^{25,26,79}$ reviewed in Falk et al. (2014). ${ }^{64}$ Based on the results from the present study, the complexity of DNA damage influences the speed of DSB recognition or $\gamma \mathrm{H} 2 \mathrm{AX} / 53 \mathrm{BP} 1$ foci formation.

In summary, different particles with similar LET and energy values induce DSB damage with different microdosimetric (spatiotemporal) characteristics that are correlated with the efficiency of DSB repair and cell survival. We propose that the extent and character of DSBs induced by radiation types with similar LET and energy values critically depend on the particle track core diameter. However, the physical quality of the particle that primarily dictates this diameter requires further study. Hence, not only the physical characteristics of radiation types but also the spatiotemporal features and behaviours of induced DNA damage should be considered in future radiotherapy studies and investigations of the effects/risks of space missions.

\section{Conflicts of interest}

The authors have no conflicts of interest to declare.

\section{Acknowledgements}

The authors thank Y. G. Teterev and A. A. Bezbakh from the Flerov Laboratory of Nuclear Reactions (JINR) and
G. N. Timoshenko and V. A. Krylov from the Laboratory of Radiation Biology (JINR) for exposing the cells to radiation with accelerated heavy ions using the U400M cyclotron and V. N. Gaevskiy from the Dzhelepov Laboratory of Nuclear Problems (JINR) for performing the gamma irradiation using the Rokus-M unit.

The work was supported by grants from the Czech Republic to the Joint Institute for Nuclear Research, Dubna (Projects of the Czech Plenipotentiary and the $3+3$ Projects), the Czech Science Foundation (project 16-12454S), and the Ministry of Health of the Czech Republic (AZV grant no. 16-29835A, all rights reserved).

\section{References}

1 S. Moore, F. K. T. Stanley and A. A. Goodarzi, DNA Repair, 2014, 17, 64-73.

2 E. Sage and N. Shikazono, Free Radicals Biol. Med., 2016, 107, 125-135.

3 Z. Nikitaki, V. Nikolov, I. V. Mavragani, E. Mladenov, A. Mangelis, D. A. Laskaratou, G. I. Fragkoulis, C. E. Hellweg, O. A. Martin, D. Emfietzoglou, V. I. Hatzi, G. I. Terzoudi, G. Iliakis and A. G. Georgakilas, Free Radical Res., 2016, 50, S64-S78.

4 R. Okayasu, Int. J. Cancer, 2012, 130, 991-1000.

5 F. A. Cucinotta, H. Nikjoo and D. T. Goodhead, Radiat. Res., 2000, 153, 459-468.

6 D. M. Sridharan, L. J. Chappell, M. K. Whalen, F. A. Cucinotta and J. M. Pluth, Radiat. Res., 2015, 184, 105-119.

7 D. T. Goodhead, Radiat. Prot. Dosim., 2015, 166, 276-281.

8 M. Suzuki, C. Tsuruoka, T. Kanai, T. Kato, F. Yatagai and M. Watanabe, Biol. Sci. Space., 2003, 17, 302-306.

9 C. Tsuruoka, M. Suzuki and K. Fujitaka, Biol. Sci. Space, 2004, 18, 188-189.

10 T. Takatsuji, I. Yoshikawa and M. S. Sasaki, J. Radiat. Res., 1999, 40, 59-69.

11 M. Niemantsverdriet, M.-J. van Goethem, R. Bron, W. Hogewerf, S. Brandenburg, J. A. Langendijk, P. van Luijk and R. P. Coppes, Int. J. Radiat. Oncol., Biol., Phys., 2012, 83, 1291-1297.

12 H. Tauchi, S. Endo, K. Eguchi-Kasai, Y. Furusawa, M. Suzuki, S. Matsuura, K. Ando, N. Nakamura, S. Sawada and K. Komatsu, J. Radiat. Res., 1999, 40 Suppl, 45-52.

13 Y. Kazama, K. Ishii, T. Hirano, T. Wakana, M. Yamada, S. Ohbu and T. Abe, Plant J., 2017, 38, 42-49.

14 P. Bláha, N. A. Koshlan, I. V. Koshlan, D. V. Petrova, Y. V. Bogdanova, R. D. Govorun, V. Múčka and E. A. Krasavin, Mutat. Res., Fundam. Mol. Mech. Mutagen., 2017, 803-805, 35-41.

15 F. Yatagai, Biol. Sci. Space, 2004, 18, 224-234.

16 R. M. Anderson, D. L. Stevens, N. D. Sumption, K. M. S. Townsend, D. T. Goodhead and M. A. Hill, Radiat. Res., 2007, 167, 541-550. 
17 H. A. Foster, G. Estrada-Girona, M. Themis, E. Garimberti, M. A. Hill, J. M. Bridger and R. M. Anderson, Mutat. Res., Genet. Toxicol. Environ. Mutagen., 2013, 756, 66-77.

18 S. Ritter, E. Nasonova and E. Gudowska-Novak, Int. J. Radiat. Biol., 2002, 78, 191-202.

19 I. Testard, B. D. X. Utrillau and L. Sabatier, Int. J. Radiat. Biol., 1997, 72, 423-433.

20 K. A. M. Suzuki, Y. Kase, H. Yamaguchi and T. Kanai, Int. J. Radiat. Oncol., Biol., Phys., 2000, 48, 241-250.

21 J. Kiefer, P. Schmidt and S. Koch, Radiat. Res., 2001, 156, 607-611.

22 M. C. Joiner, in Basic Clinical Radiobiology, ed. M. Joiner and A. van der Kogel, Hodder Arnold, 338 Euston Road, London, NW1 3BH, Great Britain, 4th edn, 2009, pp. 68-77.

23 J. A. Aten, J. Stap, P. M. Krawczyk, C. H. van Oven, R. A. Hoebe, J. Essers and R. Kanaar, Science, 2004, 303, 92-95.

24 M. Falk, E. Lukasova and S. Kozubek, Mutat. Res., 2010, 704, 88-100.

25 M. J. Kruhlak, A. Celeste, G. Dellaire, O. FernandezCapetillo, W. G. Müller, J. G. McNally, D. P. Bazett-Jones and A. Nussenzweig, J. Cell Biol., 2006, 172, 823-834.

26 B. Jakob, J. Splinter and G. Taucher-Scholz, Radiat. Res., 2009, 171, 405-418.

27 E. P. Rogakou, D. R. Pilch, A. H. Orr, V. S. Ivanova and W. M. Bonner, J. Biol. Chem., 1998, 273, 5858-5868.

28 E. P. Rogakou, C. Boon, C. Redon and W. M. Bonner, J. Cell Biol., 1999, 146, 905-916.

29 B. Jakob, M. Scholz and G. Taucher-Scholz, Radiat. Res., 2003, 159, 676-684.

30 J. Saha, P. Wilson, P. Thieberger, D. Lowenstein, M. Wang and F. A. Cucinotta, Radiat. Res., 2014, 182, 282-291.

31 A. Asaithamby, N. Uematsu, A. Chatterjee, M. D. Story, S. Burma and D. J. Chen, Radiat. Res., 2008, 169, 437-446.

32 G. Du, G. A. Drexler, W. Friedland, C. Greubel, V. Hable, R. Krücken, A. Kugler, L. Tonelli, A. A. Friedl and G. Dollinger, Radiat. Res., 2011, 176, 706-715.

33 O. A. Sedelnikova, D. R. Pilch, C. Redon and W. M. Bonner, Cancer Biol. Ther., 2003, 2, 233-235.

34 S. V. Costes, A. Ponomarev, J. L. Chen, D. Nguyen, F. A. Cucinotta and M. H. Barcellos-Hoff, PLoS Comput. Biol., 2007, 3, e155.

35 R. Lopez Perez, G. Best, N. H. Nicolay, C. Greubel, S. Rossberger, J. Reindl, G. Dollinger, K.-J. Weber, C. Cremer and P. E. Huber, FASEB J., 2016, 30, 2767-2776.

36 Y. Zhang, G. Máté, P. Müller, S. Hillebrandt, M. Krufczik, M. Bach, R. Kaufmann, M. Hausmann and D. W. Heermann, PLoS One, 2015, 10, e0128555.

37 M. Falk, Biophys. J., 2016, 110, 872-873.

38 Y. Lorat, C. U. Brunner, S. Schanz, B. Jakob, G. TaucherScholz and C. E. Rübe, DNA Repair, 2015, 28, 93-106.

39 Y. Lorat, S. Timm, B. Jakob, G. Taucher-Scholz and C. E. Rübe, Radiother. Oncol., 2016, 121, 154-161.

40 C. E. Rübe, Y. Lorat, N. Schuler, S. Schanz, G. Wennemuth and C. Rübe, DNA Repair, 2011, 10, 427-437.

41 F. Tommasino, E. Scifoni and M. Durante, Int. J. Part. Ther., 2015, 2, 428-438.
42 E. C. Halperin, Lancet Oncol., 2006, 7, 676-685.

43 M. Wang, J. Saha and F. A. Cucinotta, Mutat. Res., Genet. Toxicol. Environ. Mutagen., 2013, 756, 108-114.

44 A. L. Ponomarev and F. A. Cucinotta, Radiat. Meas., 2006, 41, 1075-1079.

45 W. Friedland, E. Schmitt, P. Kundrát, M. Dingfelder, G. Baiocco, S. Barbieri and A. Ottolenghi, Sci. Rep., 2017, 7, 45161.

46 W. E. Wilson and T. L. Criswell, Adv. Space Res., 1986, 6, 75-81.

47 N. Hamada, J. Radiat. Res., 2009, 50, 1-9.

48 B. Jakob, J. Splinter, S. Conrad, K.-O. Voss, D. Zink, M. Durante, M. Lobrich and G. Taucher-Scholz, Nucleic Acids Res., 2011, 39, 6489-6499.

49 A. A. Bezbakh, V. B. Zager, G. Kaminski, A. I. Krylov, V. A. Krylov, Y. G. Teterev and G. N. Timoshenko, Phys. Part. Nucl. Lett., 2013, 10, 175-178.

50 O. B. Tarasov and D. Bazin, Nucl. Instrum. Methods Phys. Res., Sect. B, 2016, 376, 185-187.

51 P. Matula, M. Maška, O. Daněk, P. Matula and M. Kozubek, in IEEE International Symposium on Biomedical Imaging, IEEE, 2009, pp. 1138-1141.

52 J. M. Daley and P. Sung, Mol. Cell. Biol., 2014, 34, 13801388.

53 S. Pellegrino, J. Michelena, F. Teloni, R. Imhof and M. Altmeyer, Cell Rep., 2017, 19, 1819-1831.

54 A. J. Nakamura, V. A. Rao, Y. Pommier and W. M. Bonner, Cell Cycle, 2010, 9, 389-397.

55 W. S. Rasband, ImageJ, U. S. National Institutes of Health, Bethesda, Maryland, USA, 1997-2016, https://imagej.nih. gov/ij/.

56 T. R. Jones, I. Kang, D. B. Wheeler, R. A. Lindquist, A. Papallo, D. M. Sabatini, P. Golland and A. E. Carpenter, BMC Bioinf., 2008, 9, 482.

57 A. Chatterjee and W. R. Holley, Adv. Radiat. Biol., 1993, 17, 181-226.

58 A. Asaithamby and D. J. Chen, Mutat. Res., Fundam. Mol. Mech. Mutagen., 2011, 711, 87-99.

59 M. Hofer, M. Falk, D. Komůrková, I. Falková, A. Bačíková, B. Klejdus, E. Pagáčová, L. Štefančíková, L. Weiterová, K. J. Angelis, S. Kozubek, L. Dušek and Š. Galbavý, J. Med. Chem., 2016, 59, 3003-3017.

60 S. Tenhumberg, E. Gudowska-Nowak, E. Nasonova and S. Ritter, Int. J. Radiat. Biol., 2007, 83, 501-513.

61 N. I. Nakajima, H. Brunton, R. Watanabe, A. Shrikhande, R. Hirayama, N. Matsufuji, A. Fujimori, T. Murakami, R. Okayasu, P. Jeggo and A. Shibata, PLoS One, 2013, 8, e70107.

62 D. M. Sridharan, A. Asaithamby, S. M. Bailey, S. V. Costes, P. W. Doetsch, W. S. Dynan, A. Kronenberg, K. N. Rithidech, J. Saha, a. M. Snijders, E. Werner, C. Wiese, F. A. Cucinotta and J. M. Pluth, Radiat. Res., 2015, 183, 1-26.

63 D. T. Goodhead, J. Radiat. Res., 1999, 40(Suppl), 1-13.

64 M. Falk, M. Hausmann, E. Lukasova, A. Biswas, G. Hildenbrand, M. Davidkova, E. Krasavin, Z. Kleibl, 
I. Falkova, L. Jezkova, L. Stefancikova, J. Sevcik, M. Hofer, A. Bacikova, P. Matula, A. Boreyko, J. Vachelova, A. Michaelidesova and S. Kozubek, Crit. Rev. Eukaryotic Gene Expression, 2014, 24, 225-247.

65 S. V. Costes, A. Boissière, S. Ravani, R. Romano, B. Parvin and M. H. Barcellos-Hoff, Radiat. Res., 2006, 165, 505-515.

66 F. Antonelli, A. Campa, G. Esposito, P. Giardullo, M. Belli, V. Dini, S. Meschini, G. Simone, E. Sorrentino, S. Gerardi, G. A. P. Cirrone and M. A. Tabocchini, Radiat. Res., 2015, 183, 417-431.

67 M. Falk, M. Hausmann, E. Lukasova, A. Biswas, G. Hildenbrand, M. Davidkova, E. Krasavin, Z. Kleibl, I. Falkova, L. Jezkova, L. Stefancikova, J. Sevcik, M. Hofer, A. Bacikova, P. Matula, A. Boreyko, J. Vachelova, A. Michaelidesova and S. Kozubek, Crit. Rev. Eukaryotic Gene Expression, 2014, 24, 205-223.

68 J. Reindl, G. A. Drexler, S. Girst, C. Greubel, C. Siebenwirth, S. E. Drexler, G. Dollinger and A. A. Friedl, Phys. Biol., 2015, 12, 66005.

69 Y. Lorat, S. Schanz, N. Schuler, G. Wennemuth, C. Rübe and C. E. Rübe, PLoS One, 2012, 7, e38165.

70 Y. Lorat, S. Schanz and C. E. Rübe, Clin. Cancer Res., 2016, 22, 5300-5311.

71 M. Winey, J. B. Meehl, E. T. O’Toole and T. H. Giddings, Mol. Biol. Cell, 2014, 25, 319-323.

72 A. Bolzer, G. Kreth, I. Solovei, D. Koehler, K. Saracoglu, C. Fauth, S. Müller, R. Eils, C. Cremer, M. R. Speicher and T. Cremer, PLoS Biol., 2005, 3, e157.

73 D.-H. Kim, B. Li, F. Si, J. M. Phillip, D. Wirtz and S. X. Sun, J. Cell Sci., 2016, 129, 457-457.

74 J. C. Waters, J. Cell Biol., 2009, 185, 1135-1148.

75 M. Hausmann, N. Ilić, G. Pilarczyk, J.-H. Lee, A. Logeswaran, A. Borroni, M. Krufczik, F. Theda, N. Waltrich, F. Bestvater, G. Hildenbrand, C. Cremer and M. Blank, Int. J. Mol. Sci., 2017, 18, 2066.

76 M. Krufczik, A. Sievers, A. Hausmann, J.-H. Lee, G. Hildenbrand, W. Schaufler and M. Hausmann, Int. J. Mol. Sci., 2017, 18, 1005.

77 J. P. Eberle, A. Rapp, M. Krufczik, M. Eryilmaz, M. Gunkel, H. Erfle and M. Hausmann, Methods Mol. Biol., 2017, 1663, $1-13$.

78 M. Bach, C. Savini, M. Krufczik, C. Cremer, F. Rösl and M. Hausmann, Int. J. Mol. Sci., 2017, 18, 1726.

79 M. Falk, E. Lukášová and S. Kozubek, Biochim. Biophys. Acta, Mol. Cell Res., 2008, 1783, 2398-2414.

80 S. Kozubek, E. Lukásová, P. Jirsová, I. Koutná, M. Kozubek, A. Ganová, E. Bártová, M. Falk and R. Paseková, Chromosoma, 2002, 111, 321-331.
81 M. Falk, E. Lukasova, B. Gabrielova, V. Ondrej and S. Kozubek, Biochim. Biophys. Acta, Mol. Cell Res., 2007, 1773, 1534-1545.

82 D. Deckbar, P. A. Jeggo and M. Löbrich, Crit. Rev. Biochem. Mol. Biol., 2011, 46, 271-283.

83 N. Shikazono, M. Noguchi, K. Fujii, A. Urushibara and A. Yokoya, J. Radiat. Res., 2009, 50, 27-36.

84 S. K. Singh, M. Wang, C. Staudt and G. Iliakis, Nucleic Acids Res., 2011, 39, 8416-8429.

85 L. Ježková, M. Falk, I. Falková, M. Davídková, A. Bačíková, L. Štefančíková, J. Vachelová, A. Michaelidesová, E. Lukášová, A. Boreyko, E. Krasavin and S. Kozubek, Appl. Radiat. Isot., 2014, 83, 128-136.

86 M. Falk, E. Lukášová, L. Štefančíková, E. Baranová, I. Falková, L. Ježková, M. Davídková, A. Bačíková, J. Vachelová, A. Michaelidesová and S. Kozubek, Appl. Radiat. Isot., 2014, 83, 177-185.

87 Y. Ziv, D. Bielopolski, Y. Galanty, C. Lukas, Y. Taya, D. C. Schultz, J. Lukas, S. Bekker-Jensen, J. Bartek and Y. Shiloh, Nat. Cell Biol., 2006, 8, 870-876.

88 I. Chiolo, A. Minoda, S. U. Colmenares, A. Polyzos, S. V. Costes and G. H. Karpen, Cell, 2011, 144, 732744.

89 I. Chiolo, J. Tang, W. Georgescu and S. V. Costes, Mutat. Res., Fundam. Mol. Mech. Mutagen., 2013, 750, 56-66.

90 W. Friedland, P. Jacob, H. G. Paretzke and T. Stork, Radiat. Res., 1998, 150, 170.

91 W. Friedland, P. Jacob, H. G. Paretzke, A. Ottolenghi, F. Ballarini and M. Liotta, Radiat. Prot. Dosimetry, 2006, 122, 116-120.

92 B. Boudaïffa, P. Cloutier, D. Hunting, M. A. Huels and L. Sanche, Science, 2000, 287, 1658-1660.

93 B. Jakob, J. Splinter, M. Durante and G. Taucher-Scholz, Proc. Natl. Acad. Sci. U. S. A., 2009, 106, 3172-3177.

94 R. M. Anderson, D. G. Papworth, D. L. Stevens, N. D. Sumption and D. T. Goodhead, Cytogenet. Genome Res., 2006, 112, 35-44.

95 M. Durante, D. Pignalosa, J. A. Jansen, X. F. Walboomers and S. Ritter, Radiat. Res., 2010, 174, 20-26.

96 G. Kraft, M. Kramer and M. Scholz, Radiat. Environ. Biophys., 1992, 31, 161-180.

97 V. Conte, P. Colautti, B. Grosswendt, D. Moro and L. De Nardo, New J. Phys., 2012, 14, 93010.

98 T. Neumaier, J. Swenson, C. Pham, A. Polyzos, A. T. Lo, P. Yang, J. Dyball, A. Asaithamby, D. J. Chen, M. J. Bissell, S. Thalhammer and S. V. Costes, Proc. Natl. Acad. Sci. U. S. A., 2012, 109, 443-448. 